\title{
Translocation of mixed lineage kinase domain-like protein to plasma membrane leads to necrotic cell death
}

\author{
Xin Chen ${ }^{1, *}$, Wenjuan $\mathrm{Li}^{1,{ }^{*}}$, Junming Ren ${ }^{1, *}$, Deli Huang ${ }^{1}$, Wan-ting $\mathrm{He}^{1}$, Yunlong Song ${ }^{1}$, Chao Yang ${ }^{1}$, \\ Wanyun $\mathrm{Li}^{1}$, Xinru Zheng ${ }^{1}$, Pengda Chen ${ }^{1}$, Jiahuai Han ${ }^{1}$ \\ ${ }^{I}$ State Key Laboratory of Cellular Stress Biology, Innovation Center for Cell Biology, School of Life Sciences, Xiamen University, Xiamen, \\ Fujian 361005, China
}

Mixed lineage kinase domain-like protein (MLKL) was identified to function downstream of receptor interacting protein 3 (RIP3) in tumor necrosis factor- $\alpha$ (TNF)-induced necrosis (also called necroptosis). However, how MLKL functions to mediate necroptosis is unknown. By reconstitution of MLKL function in $M L K L$-knockout cells, we showed that the N-terminus of MLKL is required for its function in necroptosis. The oligomerization of MLKL in TNF-treated cells is essential for necroptosis, as artificially forcing MLKL together by using the hormone-binding domain (HBD*) triggers necroptosis. Notably, forcing together the $\mathrm{N}$-terminal domain (ND) but not the C-terminal kinase domain of MLKL causes necroptosis. Further deletion analysis showed that the four- $\alpha$-helix bundle of MLKL (1-130 amino acids) is sufficient to trigger necroptosis. Both the HBD*-mediated and TNF-induced complexes of MLKL(ND) or MLKL are tetramers, and translocation of these complexes to lipid rafts of the plasma membrane precedes cell death. The homo-oligomerization is required for MLKL translocation and the signal sequence for plasma membrane location is located in the junction of the first and second $\alpha$-helices of MLKL. The plasma membrane translocation of MLKL or MLKL(ND) leads to sodium influx, and depletion of sodium from the cell culture medium inhibits necroptosis. All of the above phenomena were not seen in apoptosis. Thus, the MLKL oligomerization leads to translocation of MLKL to lipid rafts of plasma membrane, and the plasma membrane MLKL complex acts either by itself or via other proteins to increase the sodium influx, which increases osmotic pressure, eventually leading to membrane rupture.

Keywords: MLKL; necroptosis; sodium influx; RIP3; plasma membrane translocation

Cell Research (2014) 24:105-121. doi:10.1038/cr.2013.171; published online 24 December 2013

\section{Introduction}

It has been known for more than two decades that the proinflammatory cytokine tumor necrosis factor- $\alpha$ (TNF) can induce apoptosis in some cell lines and necrosis in others [1]. Apoptosis, characterized by the activation of caspases, nuclear shrinkage, membrane blebbing and formation of apoptotic bodies, was once considered the sole form of programmed cell death. In contrast, necrotic cell death has been described as an uncontrolled and nonspecific mode of cell death, with features of swelling of

*These three authors contributed equally to this work.

Correspondence: Jiahuai Han

E-mail: jhan@xmu.edu.cn; jhan@scripps.edu

Received 18 November 2013; revised 28 November 2013; accepted 28

November 2013; published online 24 December 2013 cellular organelles, loss of cell membrane integrity and subsequent leakage of cell contents. However, growing evidence has unambiguously demonstrated that certain types of necrosis are tightly controlled by intrinsic cellular programs, and a receptor interacting protein 1 (RIP1)dependent necrosis was named "necroptosis" [2-4]. The most pertinent information regarding necroptosis was derived from studies of TNF-induced necroptosis. Upon TNF stimulation, the cytosolic portion of TNF receptor 1 (TNFR1) recruits several adaptor proteins to form a signaling complex termed complex I, which contains TRADD, RIP1, TRAF2/5, LUBAC and cIAP1/2 [5]. Complex I serves as a platform for the recruitment of downstream kinases and effector proteins to initiate the activation of NF- $\mathrm{NB}$ and mitogen-activated protein kinases $[6,7]$. The internalization of ligand-bound TNFR1 
is accompanied by the formation of complex II containing deubiquitinated RIP1, caspase- 8 and the adaptors TRADD and FADD [8]. Complex II can trigger apoptosis or convert to a necroptosis-inducing complex called necrosome when RIP3 is present [9-12]. RIP1 and RIP3 become phosphorylated in the necrosome and the phosphorylation of RIP3 leads to the recruitment of a mixed lineage kinase domain-like protein (MLKL) [13, 14]. A recent study revealed that MLKL is phosphorylated by RIP3 in its kinase-like domain, and the phosphorylation of MLKL is required for TNF-induced necroptosis [13]. Like RIP1 and RIP3, MLKL is indispensable for necroptosis; however, how MLKL mediates necroptotic downstream signaling of necrosome is still unclear. Because of the potential of RIP1 and RIP3 to form amyloid aggregates, the requirement of the formation of supramolecular amyloidal fibrils by RIP1, RIP3 and MLKL in necroptosis was proposed [15]. But the functional requirement of this supramolecular complex in necroptosis has not been demonstrated yet.

Morphologically, either apoptosis or necroptosis is usually coupled with the changes of cell volume [16]. Unlike plant cells having mechanically resistant cell walls, mammalian cells possess a diverse repertoire of ion channels and pumps to maintain ion gradients, particularly $\mathrm{K}^{+}, \mathrm{Cl}^{-}$and $\mathrm{Na}^{+}$, thereby establishing a relatively constant cell volume [17]. Among these ion channels and pumps, $\mathrm{Na}^{+} / \mathrm{K}^{+}$-ATPase is the major determinant in maintaining osmotic gradients across the cell membrane [18]. Under physiological conditions, the excess sodium will be pumped out and extracellular potassium will be pumped in, therefore, creating an intracellular environment with high $\mathrm{K}^{+}(\sim 140 \mathrm{mM})$ and low $\mathrm{Na}^{+}(\sim 10 \mathrm{mM})$ [19]. Cell shrinkage is an early hallmark of apoptosis, and has been demonstrated to correlate with increasing $\mathrm{K}^{+}$and $\mathrm{Cl}^{-}$efflux and activation of $\mathrm{K}^{+}$channels [20]. In a particular case, high extracellular $\mathrm{K}^{+}$can block $\mathrm{K}^{+}$efflux and completely prevent Fas ligation-induced apoptosis in Jurkat cells [21]. Inhibition of the $\mathrm{Na}^{+} / \mathrm{K}^{+}$-ATPase pump or $\mathrm{Ca}^{2+}$-dependent $\mathrm{K}^{+}$channel also contributes to protection against UV irradiation-induced apoptosis in HL60 cells [22]. In contrast to cell shrinkage resulting from cytoplasmic water removal in apoptosis, cell swelling that occurs during cell necroptosis is likely to be caused by increased water influx. Pyroptosis, a caspase 1-dependent cell death, shares several features with necroptosis, such as rapid plasma membrane rupture and the release of intracellular contents [23]. The underlying mechanism of pyroptosis is the plasma membrane pore formation induced by infection of Salmonella and other pathogens that dissipates cellular ionic gradients, allowing water influx, cell swelling and osmotic lysis [24-26]. The cytoprotective agent glycine blocks nonspecific ion fluxes in dying cells and thereby prevents cell swelling and lysis during pyroptosis [25, 27].

To address the execution mechanism downstream of MLKL in necroptosis, we first determined the functions of domains and regions in MLKL by reconstituting the function of MLKL in MLKL knockout (KO) cells. We show here that the N-terminus of MLKL is required for MLKL to mediate necroptotic signaling. We found that the interaction and phosphorylation of MLKL by RIP3 promotes oligomerization of MLKL, and either naturally or artificially inducing the oligomerization of MLKL leads to the translocation of MLKL complex to lipid rafts of the plasma membrane and subsequent sodium influx and membrane rupture. The MLKL complex is most likely homotetramers, and the tetramerization of the four- $\alpha$-helices in the N-terminal domain (ND) of MLKL is necessary and sufficient for plasma membrane translocation of MLKL and necroptosis. Targeting the plasma membrane by MLKL is a critical step in the execution of necrotic cell death.

\section{Results}

\section{MLKL ND is responsible for triggering necroptosis}

MLKL contains a pseudokinase domain (kinase domain) and an ND (Figure 1A). It is known that the kinase domain of MLKL is responsible for the interaction with RIP3 [13], but the function of ND is not clear, although it was speculated to be essential for the execution of downstream events in necroptosis. L929 is a murine fibroblast cell line and undergoes necroptosis in response to TNF stimulation [28]. We generated a MLKL KO L929 line and confirmed that TNF-induced necroptosis is blocked in this cell line [29]. As reconstitution of MLKL function in $M L K L \mathrm{KO}$ cells can be used as an assay to evaluate the functions of different MLKL domains, we constructed vectors to express C-terminal Flag-tagged full-length, kinase domain, ND and N-terminal 10-amino-acid deletion (MLKL(11-464)) of murine MLKL and expressed each of them at comparable levels in MLKL KO L929 cells (Figure 1A and 1B). As anticipated, TNF-induced cell death was restored in $M L K L \mathrm{KO}$ cells expressing full-length MLKL; and expression of ND or kinase domain of MLKL could not reconstitute MLKL's function in TNF-induced cell death (Figure 1C, left panel). Interestingly, 10-amino-acid deletion from the N-terminus of MLKL abolished the function of MLKL in TNF-induced cell death, demonstrating the importance of the N-terminal portion in the function of MLKL. The same results were obtained when the cells were stimulated by TNF plus pan-caspase inhibitor zVAD (Figure 1C, right panel), confirming that the cell death is necroptosis. We also used non-tagged MLKL and its mutants and obtained the 
A

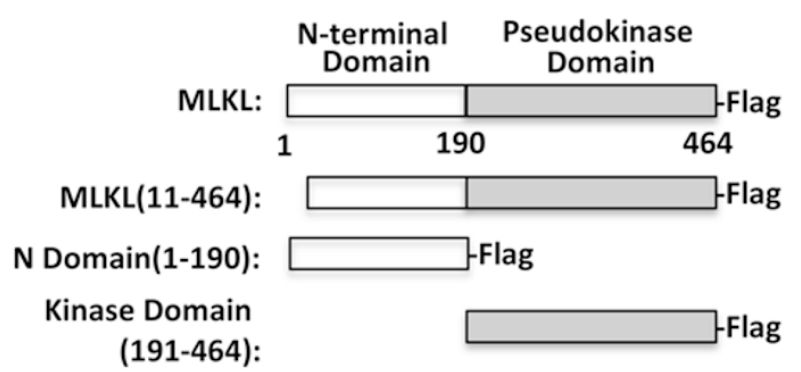

B

L929 MLKL KO

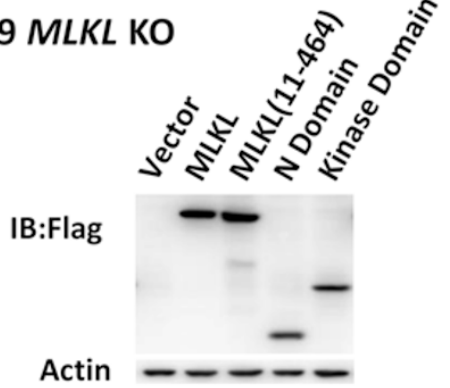

C
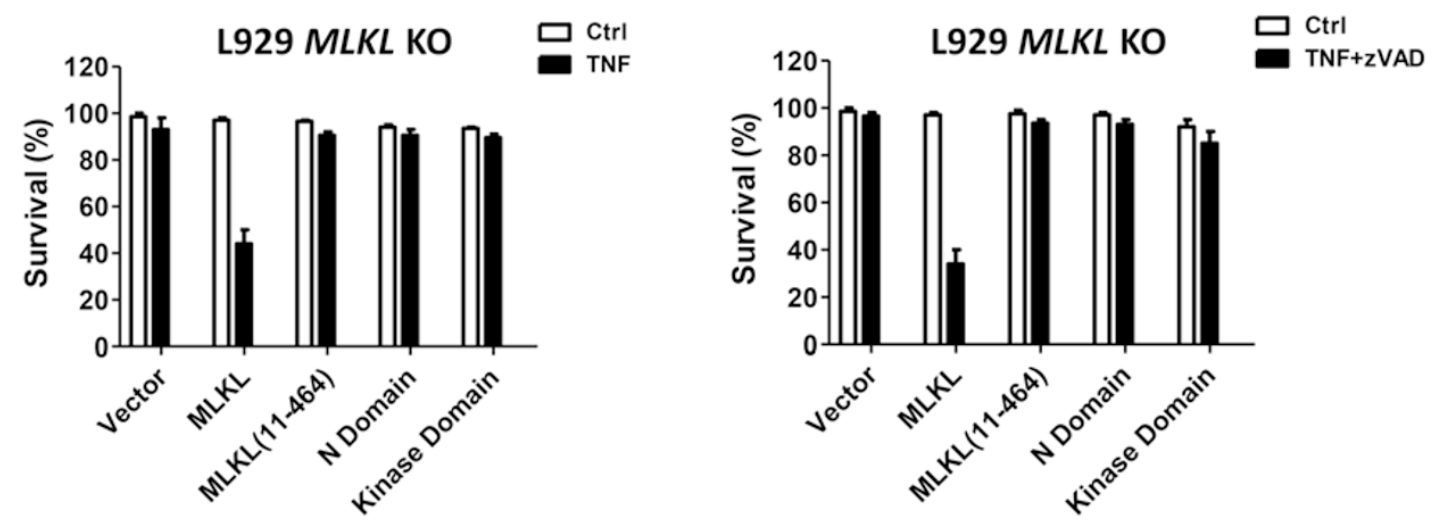

D

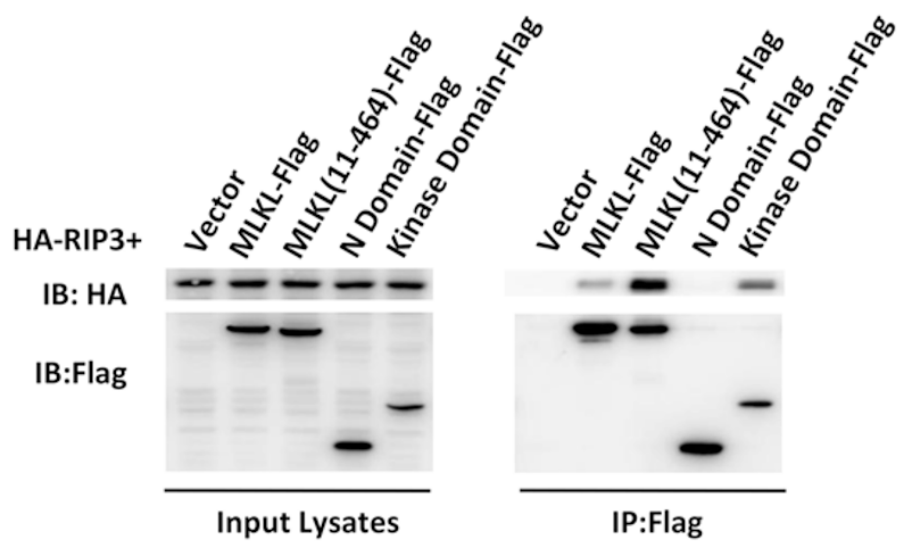

Figure 1 The N-terminus of MLKL is required for its function in necroptosis, and the N-terminal domain (ND) of MLKL is responsible for triggering necroptosis. (A) Schematic representation of full-length and truncated murine MLKL. (B) Lentiviral vector was used to express MLKL and its mutants in MLKL KO L929 cells. The expression of full-length and truncated MLKL proteins was analyzed by immunoblotting with the anti-Flag antibody $48 \mathrm{~h}$ after infection. (C) The cells described in B were treated with TNF $(10 \mathrm{ng} / \mathrm{ml})$ or TNF + ZVAD $(20 \mu \mathrm{M})$ for $12 \mathrm{~h}$ and $4 \mathrm{~h}$, respectively. Viabilities of the cells were measured by $\mathrm{PI}$ exclusion. The data represented the mean $\pm \mathrm{SD}$ of triplicates, and was representative of three independent experiments. (D) Flag-tagged full-length and truncated MLKL were cotransfected with HA-tagged RIP3 in HEK293T cells. The cell lysates were prepared $24 \mathrm{~h}$ after transfection and the immunoprecipitations were carried out by using anti-Flag antibody beads. The immunoprecipitates and cell lysates were subjected to immunoblotting with antibodies against HA and Flag.

same results (data not shown). However, expression of $\mathrm{N}$-terminal Flag-tagged MLKL in $M L K L \mathrm{KO}$ cells cannot restore TNF-induced necroptosis (data not shown), which is consistent with the data that N-terminus is im- portant for MLKL's function in necroptosis.

It was reported that human MLKL kinase domain, but not its $\mathrm{N}$ terminal portion, interacts with RIP3 [13]. We confirmed this result with the immunoprecipitation of 
murine MLKL to detect co-immunoprecipitated murine RIP3 (Figure 1D) or vice versa (Supplementary information, Figure S1). We further showed that MLKL(11-464) binds to RIP3 (Figure 1D and Supplementary information, Figure S1), even though it does not function in necroptosis (Figure 1C). Collectively, our data showed that the ND of MLKL is responsible for triggering downstream events in necroptosis and the $\mathrm{N}$-terminus of MLKL is essential for this function.

Forcing the proteins of MLKL or ND of MLKL together triggers necroptosis

As necrosome is a mega Dalton (MDa) complex [30], there should be a number of MLKL in necrosome. We therefore attempted to test whether artificially forcing MLKL molecules together would influence cell viability of L929 cells.

Oestrogen-induced homodimerization of the hormonebinding domain (HBD) of estrogen receptor can be used to induce dimer formation of a given protein fused with HBD in cells [31]. A G521R mutant of HBD (termed HBD*) that binds to a synthetic antiestrogen 4-hydroxytamoxifen (4-OHT) but not oestrogen has been used to avoid the effect of serum oestrogen in culture medium [32]. We fused HBD* to the C-terminus of MLKL and expressed it in MLKL KO L929 cells (Figure 2A and Supplementary information, Figure S2A). The addition of 4-OHT quickly induced cell death in L929 cells expressing MLKL-HBD* but not in control cells, demonstrating that $\mathrm{HBD}^{*}$-mediated interaction of MLKL is sufficient to trigger cell death (Figure 2B, left panel). Like the reconstitution experiments shown in Figure 1C, the ten N-terminus amino acids of MLKL is required for its function as 4-OHT cannot induce death of cells expressing MLKL(11-464)-HBD* (Figure 2B). Supporting the idea that ND but not the kinase domain of MLKL is responsible for downstream signaling to execute necroptosis, we found that 4-OHT also induced quick cell death in cells expressing ND-HBD* (Figure 2B, left panel) but not in cells expressing kinase domain-HBD* (data not shown). Thus, forced interaction of MLKL ND is sufficient to trigger necroptosis. We also included zVAD in the experiments and showed that it had no effect on 4-OHT-induced cell death (Figure 2B, right panel), confirming that the cell death is caspase-independent.

To confirm that 4-OHT-induced death of ND-HBD*expressing cells has necroptosis phenotype, we further analyzed the morphology of 4-OHT-induced death in ND-HBD*-expressing WT, RIP3 KO, or MLKL KO L929 cells (Supplementary information, Figure S2B, $\mathrm{S} 2 \mathrm{D}$ and $\mathrm{S} 2 \mathrm{C}$ ). The pictures in Figure $2 \mathrm{C}$ were taken at $1 \mathrm{~h}$ of $4-\mathrm{OHT}$ treatment when about $60 \%$ cells were dead in ND-HBD*-expressing WT, RIP3 KO, or MLKL
KO L929 cells (Supplementary information, Figure S3). L929 cells treated with TNF + zVAD for $4 \mathrm{~h}$ were included as a control. In both TNF + zVAD- and 4-OHT-treated samples, the dying cells became rounded and lost plasma membrane integrity. The death of ND-HBD*-expressing cells is independent of MLKL and RIP3 because deletion of $M L K L$ or RIP3 did not affect 4-OHT-induced cell death (Figure 2C and Supplementary information, Figure S3). 4-OHT-induced death of ND-HBD*-expressing cells can be reproduced in other cell types, including HeLa and Chinese hamster ovary (CHO) cells (Figure 2D and Supplementary information, Figure S2E and S2F). The expression of human MLKL(1-190)-HBD* showed the similar result (data not shown). Thus, the formation of MLKL or ND of MLKL complex can trigger necroptosis in a variety of cells.

\section{Up to four ND per 4-OHT-induced ND complex}

4-OHT-induced dimer formation is well-established in in vitro system $[31,32]$; however, the subunit number of the induced complex could be more than two if the HBD*-fused proteins are able to interact with other protein(s) through domains other than HBD*. To examine the stoichiometry of the ND complex induced by 4-OHT in our experiments, we used the SiMPull assay that combines the conventional pull-down assay with single-molecule total internal reflection fluorescence (TIRF) microscopy [33]. Cell lysate containing Flaggreen fluorescent protein (GFP)-tagged ND-HBD* was applied to polyethylene glycol (PEG)-coated coverslips. After washing away the unbound components, captured ND-HBD*-GFP-Flag molecules via surface-tethered anti-Flag antibody were visualized and ready to count the subunit number by photobleaching step analysis. Under TIRF illumination and high-sensitivity imaging, GFP alone represents only one bleaching step (data not shown), indicating that single molecule counting can be achieved in our condition. Most of ND-HBD*-GFP fluorescent spots $(\sim 90 \%)$ from untreated cells (Ctrl) showed one-step bleaching (Figure 3A), suggesting that inactive ND-HBD* is a monomer. When the cells expressing ND-HBD*-GFP were treated with 4-OHT, a fraction of the fluorescent spots became brighter and aggregated in TIRF image (Figure 3A). More and up to four bleaching steps were observed (Figure 3A), implying that ND complex may consist of up to four subunits during 4-OHTinduced necroptosis. The representative photobleaching profiles were shown in the right panels. As a control, the distribution of observed bleaching steps of HBD*-GFP was shifted from one-step $(\sim 80 \%)$ to two-step $(\sim 60 \%)$ upon addition of 4-OHT (Figure 3A, top panels), which is consistent with the fact that 4-OHT induces HBD* dimer formation as reported previously [32]. 
A

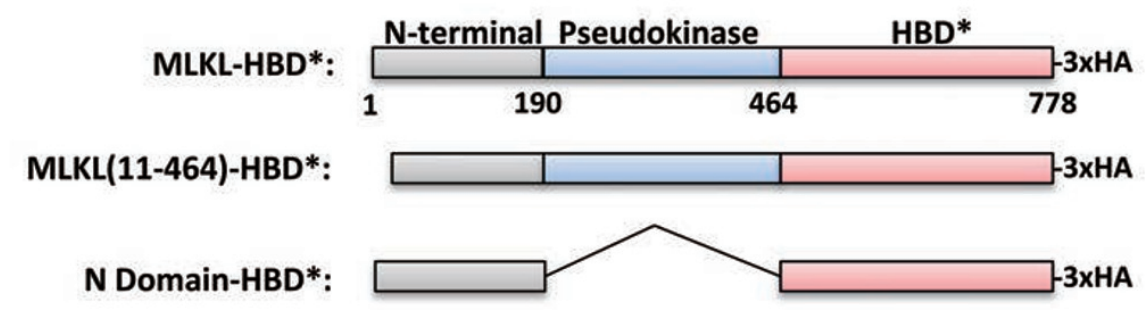

B

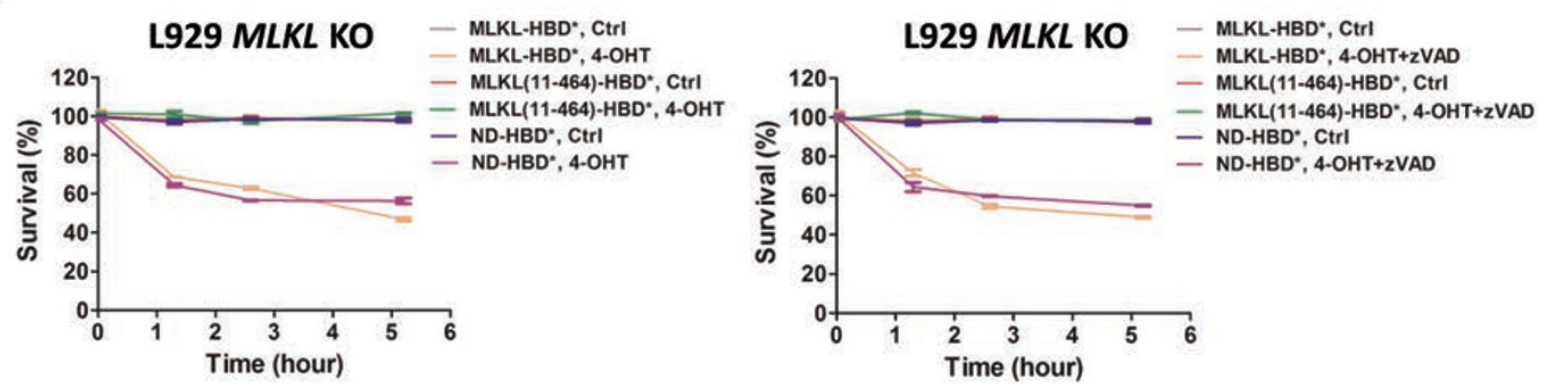

C
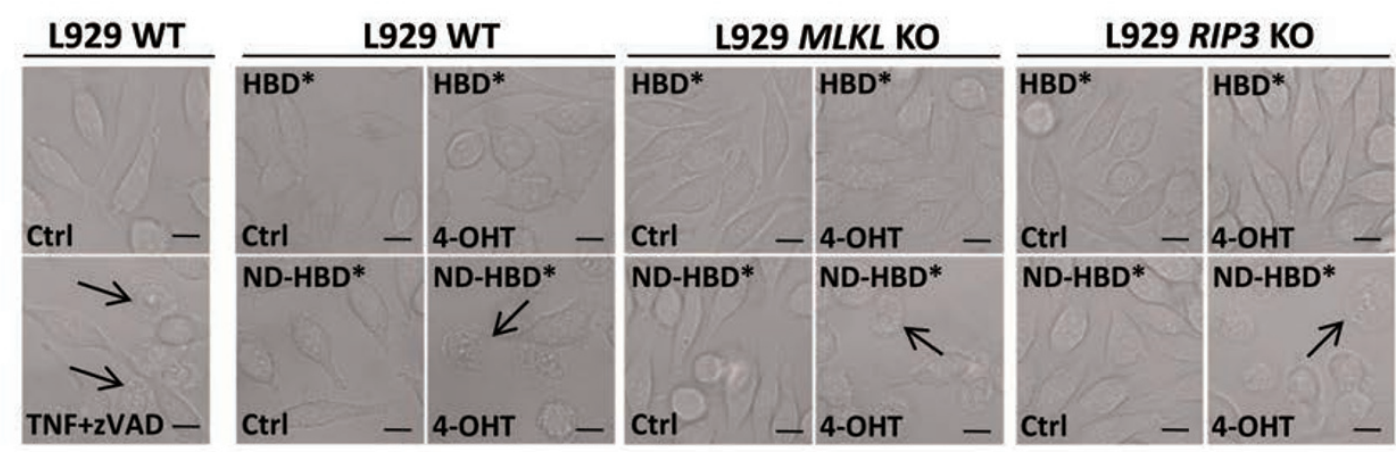

D
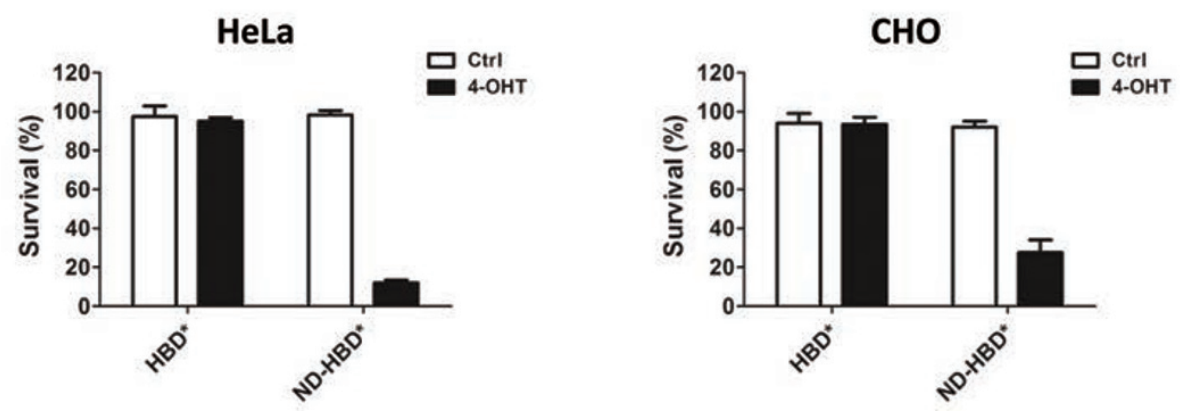

Figure 2 The HBD*-mediated interaction of MLKL or ND of MLKL can bypass RIP3 and directly induce necroptosis. (A) Schematic representation of full-length and truncated murine MLKL fused with HBD*. (B) Viabilities of MLKL KO L929 cells expressing MLKL-HBD*, MLKL(11-464)-HBD* or ND-HBD* after treatment with or without 4-OHT (1 $\mu \mathrm{M})$ or 4-OHT + zVAD $(20 \mu \mathrm{M})$ for different periods of time as indicated. (C) WT, MLKL KO and RIP3 KO L929 cells expressing HBD* or ND-HBD* were treated with or without 4-OHT $(1 \mu \mathrm{M})$ for $1 \mathrm{~h}$ and then imaged using a confocal microscope. WT L929 cells stimulated with or without TNF + zVAD $(10 \mathrm{ng} / \mathrm{ml}+20 \mu \mathrm{M})$ for $4 \mathrm{~h}$ were included as a control. The black arrows indicate necrotic cells and the scale bars represent $10 \mu \mathrm{m}$. (D) Viabilities of HeLa and CHO cells expressing HBD* or ND-HBD* after treatment with or without $4-\mathrm{OHT}(1 \mu \mathrm{M})$ for $2 \mathrm{~h}$. Data represented the mean $\pm \mathrm{SD}$ of triplicates $(\mathbf{B}, \mathbf{D})$, and were representative of three independent experiments. 
A
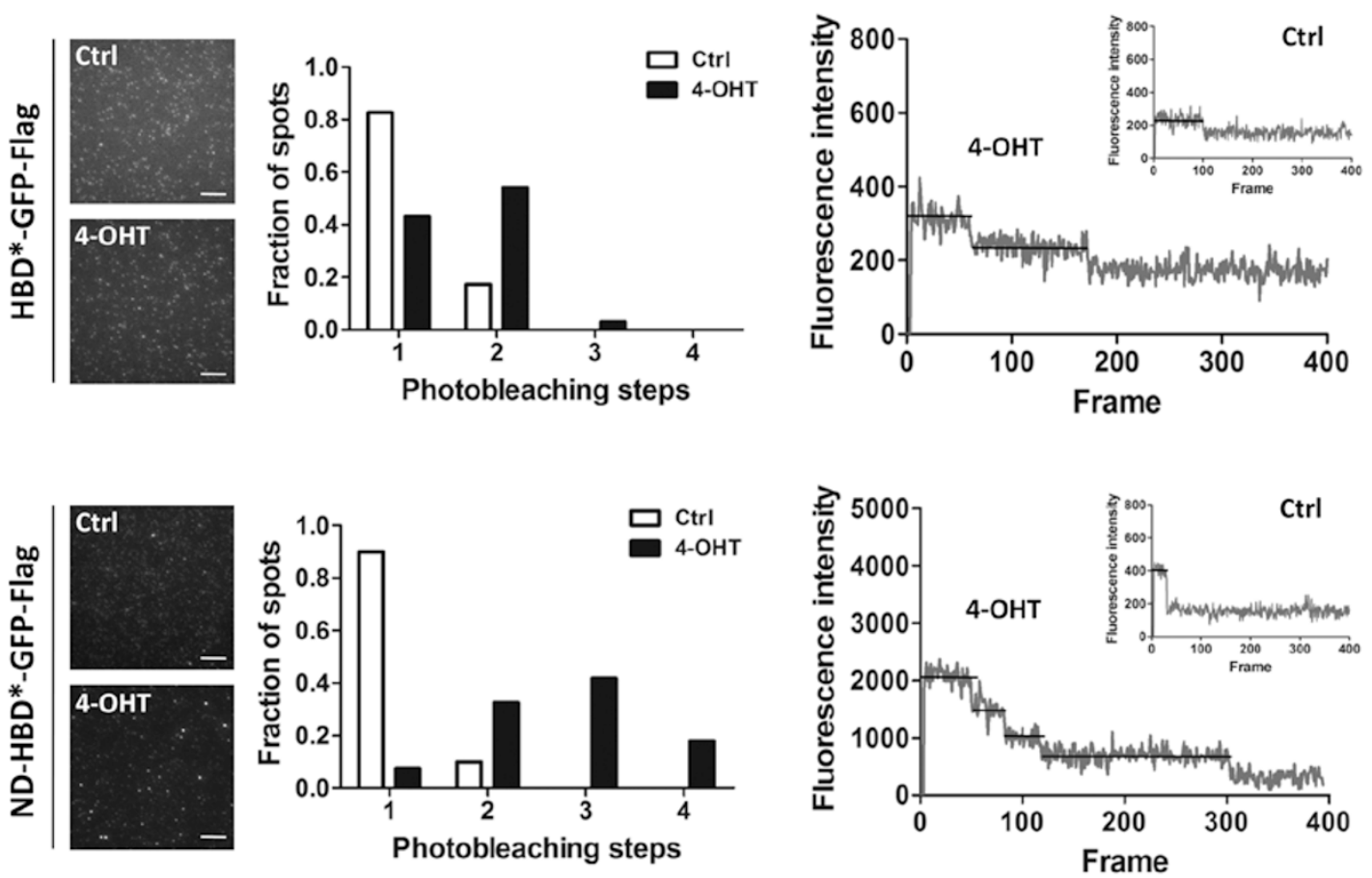

B
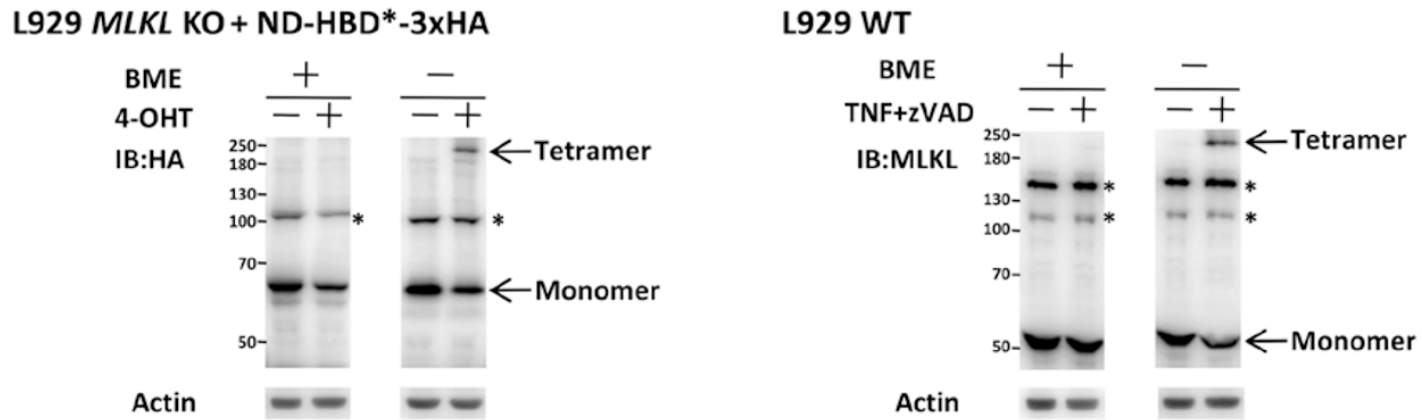

Figure 3 Both 4-OHT-induced oligomerization of ND-HBD* and TNF-induced oligomerization of MLKL produce tetramers. (A) HeLa cells were infected with a lentiviral vector encoding HBD*-GFP-Flag or ND-HBD*-GFP-Flag for $24 \mathrm{~h}$, and then treated with 4-OHT $(1 \mu \mathrm{M})$ or vehicle $(\mathrm{Ctrl})$ for $1 \mathrm{~h}$. Cells were lysed and subjected to the SiMPull assay as described in Materials and Methods. Left panels are TIRF microscopy images showing HBD*-GFP-Flag and ND-HBD*-GFP-Flag fluorescence spots on the coverslips. Each scale bar represents $5 \mu \mathrm{m}$. Examples of observed bleaching steps from HBD*-GFP-Flag- or ND-HBD*GFP-Flag-expressing cells treated with or without 4-OHT $(1 \mu \mathrm{M})$ were shown in right panels. The distribution of observed bleaching step numbers from HBD*-GFP-Flag- or ND-HBD*-GFP-Flag-expressing cells treated with or without 4-OHT (1 $\mu \mathrm{M})$ was summarized in the middle panels. (B) MLKL KO L929 cells expressing ND-HBD*-3×HA were treated with or without 4-OHT $(1 \mu \mathrm{M})$ for $1 \mathrm{~h}$, and the lysates were subjected to SDS-PAGE with or without $\beta$-mercaptoethanol (BME), and analyzed by immunoblotting using the anti-HA antibody. (C) To collect intact cells for analysis, L929 cells were stimulated with or without TNF + zVAD $(10 \mathrm{ng} / \mathrm{ml}+20 \mu \mathrm{M})$ for $3 \mathrm{~h}$, lysed and subjected to reducing or non-reducing SDS-PAGE as in B, and analyzed by immunoblotting using the anti-MLKL antibody. Arrows indicate the monomer or tetramer of ND-HBD*-3 $\times$ HA or MLKL. The asterisk denotes non-specific bands.

TNF induces formation of MLKL tetramers

To further confirm the subunit stoichiometry of the ND complex, ND-HBD*-3×HA was expressed in $M L K L$
KO L929 cells and then analyzed by reducing and nonreducing sodium dodecyl sulfate (SDS)-polyacrylamide gel electrophoresis (PAGE). ND-HBD*-3×HA from 
untreated cells was detected as monomer $(\sim 60 \mathrm{kDa})$ in both reducing and non-reducing SDS-PAGE, but part of it from 4-OHT-treated cells was detected with an apparent molecular mass of $\sim 250 \mathrm{kDa}$ on the non-reducing PAGE (Figure 3B), suggesting that 4-OHT induced the formation of ND-HBD* tetramers. This result is consistent with the data obtained by SiMPull assay (Figure $3 \mathrm{~A})$. Because the tetramers can only be detected by nonreducing PAGE, the ND-HBD* in the tetramers is most likely to be linked by disulfide bonds.

We further analyzed whether endogenous MLKL forms tetramers in L929 cells before and after TNF + zVAD treatment. As shown in Figure 3C, TNF + zVAD induced a MLKL complex with a molecular weight of $\sim 220 \mathrm{kDa}$ on non-reducing SDS-PAGE. As the molecular weight of murine MLKL is about $53 \mathrm{kDa}$ as detected in reducing SDS-PAGE, the MLKL complex detected by non-reducing gel is most likely MLKL tetramers. It is worth noting that we did not see dimer of either ND$\mathrm{HBD}^{*}$ or MLKL on the non-reducing PAGE, which indicated that there are no dimers or no disulfide bonds in the dimers. Nonetheless, tetramerization appears to be a property of MLKL protein regardless whether it was initiated by interaction with RIP3 or by forced interaction through 4-OHT-mediated HBD* dimerization.

Translocation of MLKL to the plasma membrane is associated with plasma membrane breakage

Cell fractionation has revealed that the translocation of necrosome components to membrane fractions correlates with TNF-induced cell death [29]. To further determine whether MLKL translocated to the plasma membrane, we analyzed the subcellular location of MLKL through immunofluorescence microscopy. As anti-MLKL antibodies that can detect cellular MLKL under fluorescence microscope are not available, we used $M L K L$ KO cells that were reconstituted with C-terminal $3 \times$ Flag-tagged MLKL. We have tested that the cells behaved similarly to wildtype (WT) L929 cells in TNF-induced cell death. We then treated the cells with TNF + zVAD for different periods of time and stained the cells with anti-Flag antibody for MLKL, DAPI (4',6-diamidino-2-phenylindol) for nucleus and PI (propidium iodide) for membrane breakage. The samples treated with TNF + zVAD for $3.5 \mathrm{~h}$ were used for quantification. We observed translocation of MLKL to the plasma membrane and the translocation was associated with PI-positive staining (Figure 4A). At $3.5 \mathrm{~h}$ after TNF + zVAD treatment, $\sim 30 \%$ cells were PI positive. Translocation of MLKL to plasma membrane was observed in $~ 90 \%$ PI-positive cells. The lacking of plasma membrane MLKL staining in the other $10 \%$ PI-positive cells is not due to lack of MLKL translocation, but to the release of cellular contents including MLKL to medium after plasma membrane rupture, and thus very little or no MLKL staining can be seen on these cells. There were PI-negative cells in which the MLKL had translocated to the plasma membrane, suggesting that plasma membrane breakage is likely to result from the translocation of MLKL to plasma membrane.

We then analyzed the cell death in ND-HBD*expressing cells. As 4-OHT-induced cell death is quicker than TNF-induced cell death, we chose the samples treated with 4-OHT for $1 \mathrm{~h}$ to do the quantification. As what was observed in TNF + zVAD-treated cells, 4-OHT induced translocation of ND-HBD* to the plasma membrane, and the translocation of ND-HBD* was associated with the PI-positive staining (Figure 4B). These data demonstrate that formation of MLKL complex results in MLKL translocation to the plasma membrane. The translocation of MLKL to the plasma membrane is likely to be an essential step of the late breakage of the plasma membrane.

To further confirm the plasma membrane location of ND-HBD* after 4-OHT treatment, we employed TIRF microscopy. HeLa cells stably expressing either HBD*GFP or ND-HBD*-GFP were cultured on coverslips and TIRF images were acquired as previously described [34, 35]. Because the evanescent wave illumination used in the TIRF mode decays exponentially with the distance from the water-glass interface, only fluorophores nearest the glass (TIRF zone) are selectively excited. HBD*GFP is believed to diffuse freely in the cytosol of cell, and a small portion in the vicinity of plasma membrane was also observed under TIRF illumination (Figure 4C). The addition of 4-OHT had no obvious impact on the signal distribution of HBD*-GFP. However, ND-HBD*GFP was detected as diffraction-limited bright spots in 4-OHT-treated cells, but not in untreated cells (Figure $4 \mathrm{C}$ ). The structures detected in the TIRF zone had a variable fluorescence intensity that could be due to variation in the amount of ND-HBD* molecules and/or differences in the distance of ND-HBD* from plasma membrane. These TIRF images indicate that 4-OHT induces redistribution of ND-HBD*-GFP to the plasma membrane or a place proximal to plasma membrane.

As cell fractionation showed that RIP3 translocated to membrane fractions during TNF-induced cell death [29], we sought to address whether RIP3 translocates to plasma membrane as MLKL does. RIP3 KO L929 cells reconstituted with RIP3-3×Flag were fixed and imaged. The images revealed that RIP3 is uniformly diffused throughout the cytosol in the untreated cells (Figure 4D). After $3.5 \mathrm{~h}$ of treatment with TNF and zVAD, a very small fraction of RIP3 was observed to concentrate on 
A

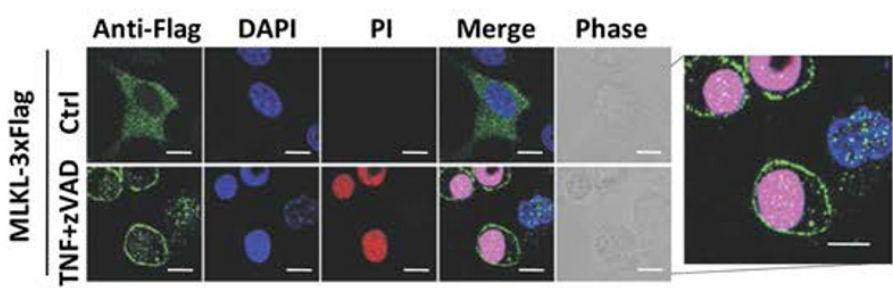

B
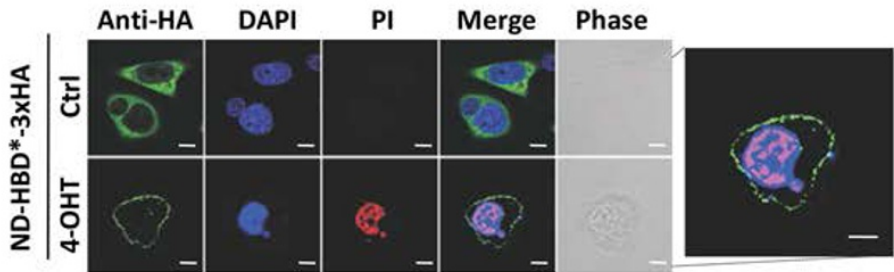

C
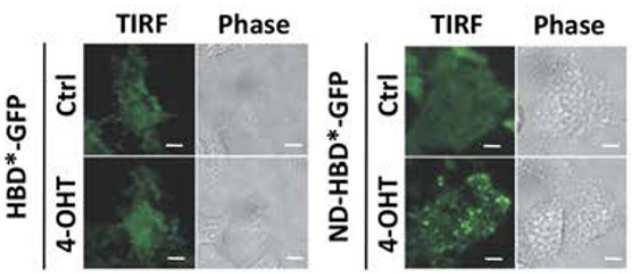

D

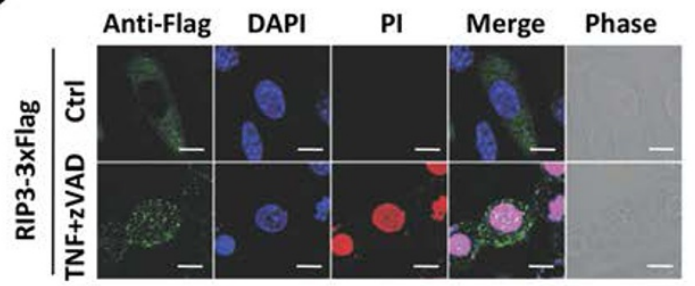

E

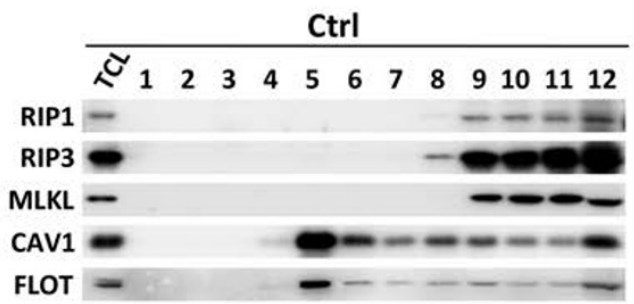

$\mathbf{F}$

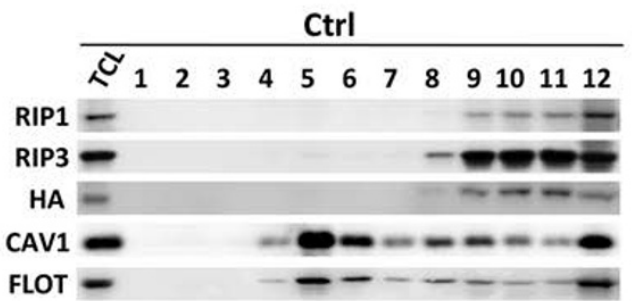

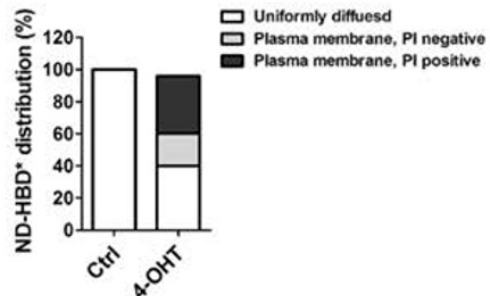

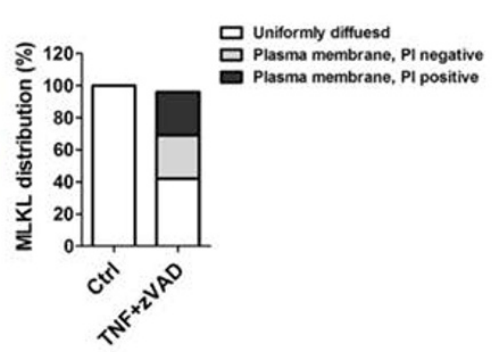

$\square$ Uniformly diftuesd

Plasma membrane, PI negativo

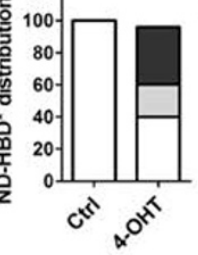

Merge Phase

TNF+ZVAD

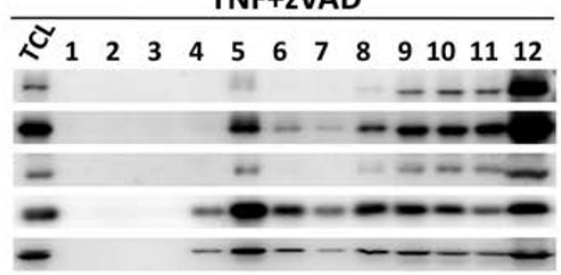

4-OHT

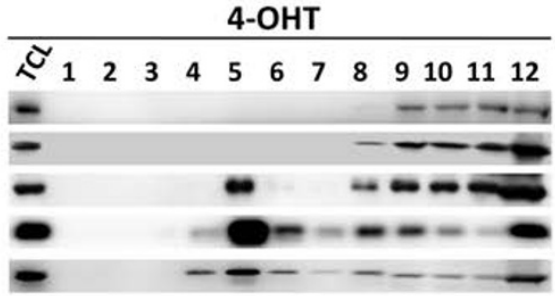

Figure 4 Both MLKL and ND-HBD* translocated to the plasma membrane and were enriched in lipid raft microdomain during necroptosis. (A) MLKL KO L929 cells reconstituted with C-terminal 3×Flag-tagged MLKL were treated with or without TNF + zVAD $(10 \mathrm{ng} / \mathrm{ml}+20 \mu \mathrm{M})$ for $3.5 \mathrm{~h}$, and then immunostained for Flag and counterstained with DAPI. PI was added to culture medium before cell fixation. The merge image of MLKL-3×Flag located in the plasma membrane was zoomed in in the middle panel. The distributions of MLKL in cells are defined as three types: uniformly diffused, plasma membrane location with PI negative and plasma membrane location with PI positive. (B) HeLa cells expressing ND-HBD*-3*HA were treated with or without 4-OHT $(1 \mu \mathrm{M})$ for $1 \mathrm{~h}$ and analyzed as described in A. (C) HeLa cells expressing HBD*-GFP or ND-HBD*-GFP were stimulated with or without 4-OHT $(1 \mu \mathrm{M})$ for $1 \mathrm{~h}$ and then imaged by TIRF microscopy. (D) RIP3 KO L929 cells reconstituted with $3 \times$ Flag-tagged RIP3 were treated with or without TNF $+z V A D(10 \mathrm{ng} / \mathrm{ml}+20 \mu \mathrm{M})$ for $3.5 \mathrm{~h}$ and analyzed as described in A. (E) L929 cells were treated with or without TNF + zVAD $(10 \mathrm{ng} / \mathrm{ml}+20 \mu \mathrm{M})$ for $2 \mathrm{~h}$, and then the total cell lysates (TCL) were fractionated to isolate lipid rafts as described in Materials and Methods. TCL and the fractions collected were immunoblotted with antibodies against RIP1, RIP3 and MLKL. Lipid raft markers CAV1 and FLOT were immunoblotted to identify lipid raft fractions. (F) MLKL KO L929 cells expressing ND-HBD*-3×HA were treated with or without 4-OHT (1 $\mu \mathrm{M})$ for $1 \mathrm{~h}$. TCL were then fractionated and analyzed as described in E. Each scale bar indicates $10 \mu \mathrm{m}$. 
the plasma membrane, while most of them formed discrete punctate as previously described [13]. This result suggests that only a small portion of RIP3, if any, translocated to plasma membrane.

\section{MLKL translocated to lipid rafts in the plasma membrane during necroptosis}

Lipid raft is a specialized microdomain containing combinations of glycosphingolipids and protein receptors in plasma membrane and perhaps other parts of the cell. Because components of necrosome including MLKL go to Triton X-100-insoluble fraction of cell lysates of necroptotic cells, and because lipid rafts are also Triton X-100 insoluble, we determined whether MLKL translocated to lipid rafts. We isolated lipid rafts by a discontinuous sucrose density gradient centrifugation from lysates of Triton X-100-treated cells and used lipid raft markers CAV1 and FLOT to identify lipid raft fractions (Figure 4E). We found that necrosome components RIP1, RIP3 and MLKL were not associated with lipid rafts in untreated cells (Figure 4E, lane 5 in left panel), and TNF + zVAD stimulation induced their translocation to lipid raft fraction (Figure 4E, lane 5 in right panel). Similarly, 4-OHT treatment also led to ND-HBD* translocation to lipid raft fraction (Figure 4F). Different from TNFtreated cells, in which not only MLKL but also RIP1 and RIP3 translocated to lipid rafts, 4-OHT only induced translocation of ND-HBD*. This is consistent with the function of 4-OHT in inducing HBD* interaction. As 4-OHT treatment can effectively induce necroptosis, translocation of RIP1 and RIP3 to lipid rafts is dispensable for necroptosis. The data shown in Figure 4E and $4 \mathrm{~F}$ indicated that MLKL translocated to lipid rafts in the plasma membrane.

Translocation of an intact four-helix bundle of MLKL ND to plasma membrane is necessary and sufficient for necroptosis

Crystal structure of MLKL revealed that the ND of MLKL is a four-helix bundle followed by a two-helix linker $[36,37]$. We further generated deletions of ND from the $\mathrm{N}$ - and $\mathrm{C}$-termini of $\mathrm{ND}$, and fused them with HBD* (Figure 5A). The corresponding positions of the deletions to the four-helix bundle and the two-helix linker were shown in the right panel of Figure 5A. Deletion of $\mathrm{N}$-terminal sequences (10 amino acids and more) abolished 4-OHT-induced cell death; even the expression of these mutants was higher than that of full-length ND (Figure 5B and 5C). The two-helix linker appears to be dispensable for cell death, as 4-OHT still induced cell death in cells expressing ND(1-130) (Figure 5A and $5 B)$. Thus, the intact four- $\alpha$-helix bundle is required for necroptosis.

Interestingly, we still observed the translocation of $\mathrm{ND}(11-190)$ and $\mathrm{ND}(21-190)$ to the plasma membrane after 4-OHT treatment (Figure 5D, left panels). In contrast, deletion of the N-terminal 40 amino acids (ND(41190)) eliminated the 4-OHT-induced ND translocation to plasma membrane (Figure 5D, top-right panels). Because amino acids 1-20 correspond to the first $\alpha$-helix in the four- $\alpha$-helix bundle, while amino acids 21-41 include the linker between the first and second $\alpha$-helices and half of the second $\alpha$-helix (Figure 5A, right panel), it seems that the sequence recognized by translocation machinery is located in the joint area of the first and second $\alpha$ - helices of MLKL.

Consistent with the data that 4-OHT induces cell death in $\mathrm{ND}(1-130)$-HBD*-expressing cells (Figure $5 \mathrm{~B}$ ), we observed plasma membrane translocation of ND(1-130)HBD* (Figure 5D, low-right panels) after 4-OHT treatment. Given the result that translocation of ND lacking the N-terminal amino acids to plasma membrane cannot cause cell death (Figure 5), we concluded that only the translocation of MLKL containing the intact four- $\alpha-$ helix bundle of ND is necessary and sufficient to trigger necroptosis.

There are hydrophobic residues in the interior side of the $\alpha$-helix bundle that may be essential for the structure of the bundle. The residues Val35, Leu38, Leu42, Leu45, Leu61, Phe64, Leu68, Ala71, Ile75, Phe78 are located either at the second or third $\alpha$-helix and make hydrophobic contacts in the four- $\alpha$-helix bundle (Supplementary information, Figure S4A, right panels). To test whether these hydrophobic residues are important for maintaining MLKL structure and function, we generated mutants by substituting each of these residues with glutamic acid (E), namely $\mathrm{ND}(\mathrm{V} 35 \mathrm{E} / \mathrm{L} 38 \mathrm{E}$ or $\mathrm{L} 42 \mathrm{E} / \mathrm{L} 45 \mathrm{E}$ or $\mathrm{L} 61 \mathrm{E} / \mathrm{F} 64 \mathrm{E}$ or L68E/A71E or I75E/F78E)-HBD*. All these mutations abolished the plasma membrane translocation of NDHBD* upon 4-OHT stimulation (Supplementary information, Figure S4A) and eliminated ND's ability to mediate necroptosis in MLKL KO L929 cells (Supplementary information, Figure S4B). The expression of all mutants was almost equal to that of ND-HBD* (Supplementary information, Figure S4C). Therefore, we suggested that these hydrophobic residues are critical for ND-HBD* to translocate to plasma membrane and induce necroptosis.

Translocation of MLKL to the plasma membrane leads to sodium influx and cell death

As the morphology of necroptotic cell death is cell swelling and plasma membrane disruption, we examined whether intracellular ion homeostasis is maintained during cell necroptosis. To monitor the intracellular sodium 
A

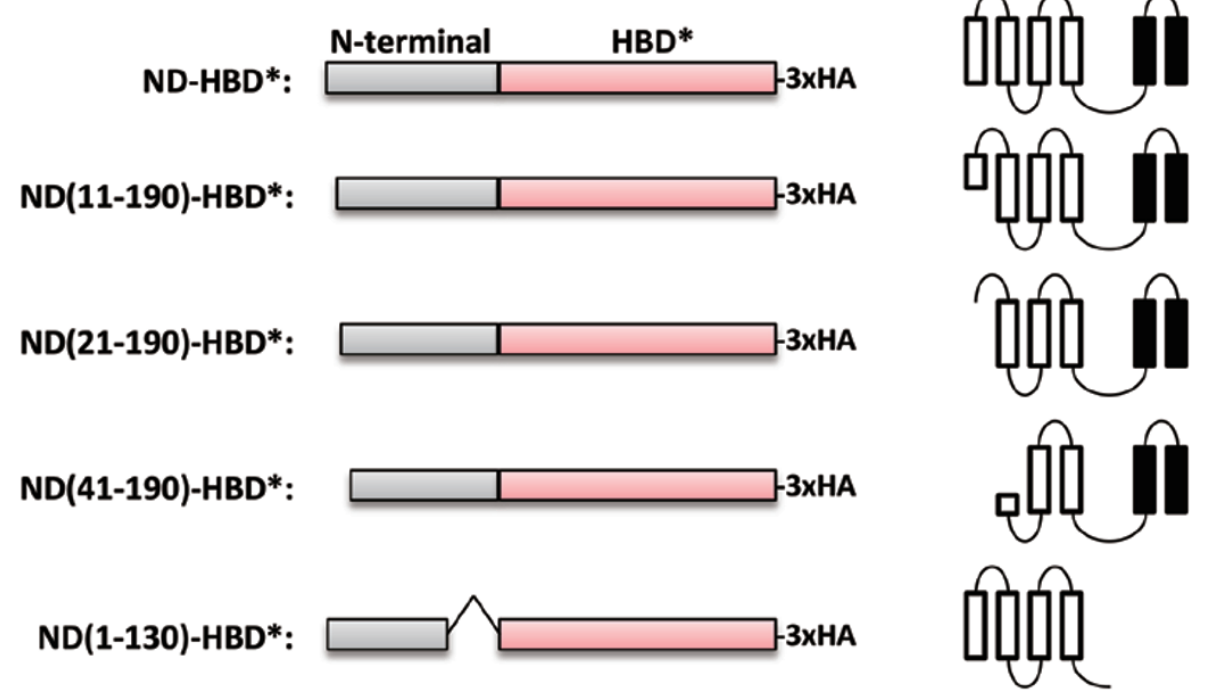

B

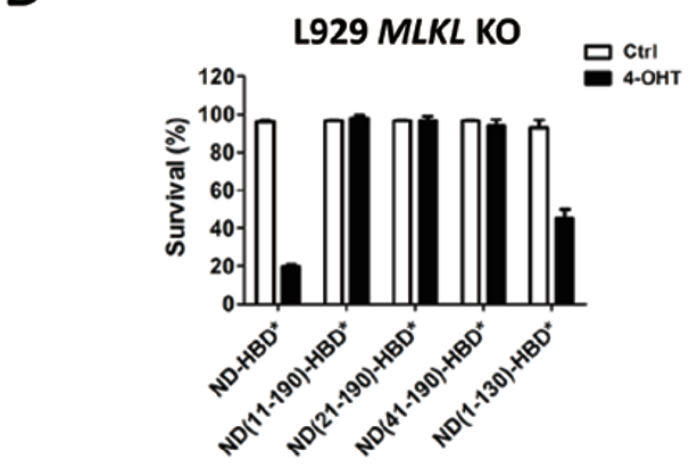

C

L929 MLKL KO

D
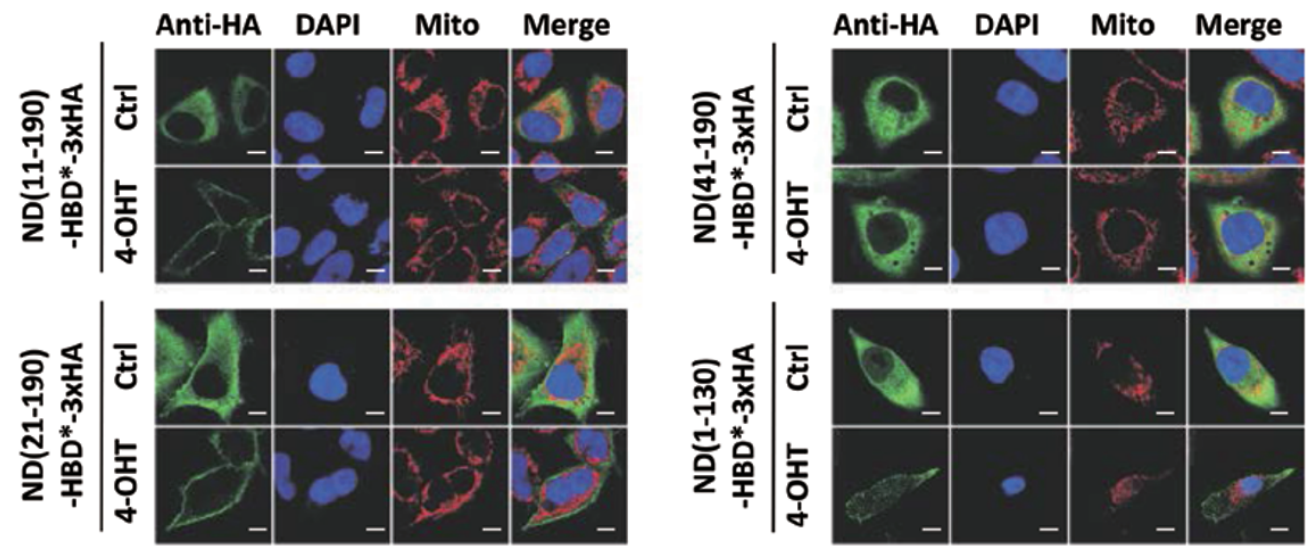

Figure 5 Translocation of the N-terminal four- $\alpha$-helix bundle of MLKL to plasma membrane is necessary and sufficient for triggering necroptosis, and targeting to plasma membrane is determined by the certain sequence within the $\alpha$-helix bundle. (A) Schematic representation of full-length and truncated ND-HBD*-3 $\times$ HA. The corresponding positions of the different deletions in $\alpha$-helices are shown in the right panels. (B) Viabilities of MLKL KO L929 cells expressing ND-HBD* or truncated ND-HBD* $(11-190,21-190,41-190,1-130)$ as described in $\mathbf{A}$ after stimulation with or without 4-OHT (1 $\mu \mathrm{M})$ for $2 \mathrm{~h}$. Data represented the mean \pm SD of triplicates, and were representative of three independent experiments. (C) Expression levels of ND-HBD* and its truncated forms in cells described in B were determined by immunoblotting with the anti-HA antibody. (D) MLKL KO L929 cells reconstituted with full-length or truncated ND-HBD*-3×HA were treated with or without 4-OHT $(1 \mu \mathrm{M})$ for $2 \mathrm{~h}$ and then immunostained with anti-HA antibody and counterstained with DAPI and Mitotracker Red. Each scale bar represents $10 \mu \mathrm{m}$. 
level during necroptosis, cells were loaded with CoroNa Green, which exhibits increased fluorescence emission intensity upon $\mathrm{Na}^{+}$binding. We used time-lapse fluorescence microscopy to monitor 4-OHT-stimulated NDHBD*-expressing cells. A $M L K L$ KO L929 cell line was used to avoid any influence of endogenous MLKL. Upon 4-OHT stimulation, ND-HBD* cells displayed an obvious increase of CoroNa Green fluorescence, and the fluorescence maintained for a while and then suddenly dropped when the cell membrane rupture occurred as indicated by PI uptake (Figure 6A and 6B). We then analyzed TNF + zVAD-induced necroptosis in L929 cells using the same approach. TNF + zVAD stimulation increased CoroNa Green fluorescence intensity and the green fluorescence dropped slowly for a period of time. and then rapidly reduced to background level at a time point just before PI uptake (Figure 6C and 6D). These data suggest that sodium overload causes cell lysis.

To verify that sodium influx is specifically associated with necroptosis but not apoptosis, we monitored the CoroNa Green fluorescence intensity in apoptosis. To make the comparison between cells with the closest background, we used RIP1 KO L929 cell, which undergoes quick apoptosis upon TNF treatment. Under light microscopy, apoptotic cells rapidly became rounded and detached from the substratum, shrank and underwent intense membrane blebbing (Figure 7A). We did not detect an increase of CoroNa Green fluorescence in the course of apoptosis (Figure 7A and 7B). When cell death culminated after TNF stimulation for $300 \mathrm{~min}$ as detected by PI uptake (termed secondary necrosis), the CoroNa Green fluorescence intensity decreased to background level due to dye release, and the cells became PI positive (Figure 7A and 7B). Thus, sodium influx does not increase in apoptosis and dose not play any role in apoptosis.

To test the selectivity of sodium influx during necroptosis, L929 cells were loaded with calcium indicator Fluo-4 and potassium indicator PBFI seperately. The relative fluorescence intensity of cells was monitored individually in real time. There are variations of Fluo-4 fluorescence intensity and the changes were within one fold in most of the cells during TNF $+\mathrm{zVAD}$ treatment (Figure $7 \mathrm{C})$. The level of the changes of Fluo-4 fluorescence in the course of necroptosis has no correlation with cell death. In the case of potassium, the relative fluorescence ratio of $340 / 380 \mathrm{~nm}$, which is an indicator of potassium concentration, was almost unchanged during necroptosis (Figure 7D). Therefore, sodium influx is likely to be selectively involved in necroptosis.

As intracellular sodium concentration increased during necroptosis, we tested the requirement of sodium influx for cells undergoing necroptosis. L929 cells were cultured in regular medium and sodium-free (N-methyl-Dglucamine (NMDG)) medium, respectively, and treated with TNF + zVAD. Cell viability was measured at different time points (Figure 8A). Sodium depletion in culture medium inhibited the TNF + zVAD-induced necroptosis. We also tested whether sodium depletion would protect 4-OHT-induced cell death in cells expressing NDHBD*, and found that indeed it significantly inhibited the cell death of cells expressing ND-HBD* (Figure 8B). In contrast, depletion of calcium in the medium did not affect necroptosis and removal of potassium enhanced cell death (data not shown). Importantly, TNF-induced apoptosis in RIP1 KO L929 cells was not affected by sodium depletion (Figure $8 \mathrm{C}$ ), indicating that sodium influx is selectively required for necroptosis. Moreover, we also monitored the CoroNa Green fluorescence intensity of L929 cells undergoing TNF + zVAD-induced necroptosis in the sodium-free medium. No obvious fluorescence change was observed (Figure 8D), suggesting that the increase of CoroNa Green fluorescence shown in Figure 6D was due to sodium influx. Collectively, our data showed that MLKL complex on plasma membrane functions to increase the sodium influx; the increased intracellular sodium concentration should lead to increased osmotic pressure, resulting in increased water uptake and plasma membrane rupture.

\section{Discussion}

Despite the great progresses made in recent years on the mechanisms of programmed necrosis, how cell swelling and plasma membrane rupture, the common features of necrosis, are executed is still not very clear. The discovery of the translocation of MLKL complex to plasma membrane sheds light on the execution mechanisms of programmed necrosis. Based on ours and published data, we proposed a model as follows. The recruitment of MLKL by RIP3 leads to phosphorylation of MLKL and tetramerization of MLKL. The tetramerized MLKLs translocate to lipid rafts of plasma membrane by an unknown mechanism, but certain structural features in the area between the first and second $\alpha$-helices of MLKL are required. Translocation of an intact four- $\alpha$-helix bundle to plasma membrane is necessary and sufficient for necroptosis. The MLKL complex on plasma membrane is able, either by itself or through other membrane protein(s), to increase sodium influx. The enhanced intracellular sodium concentration increases osmotic pressure, leading to water influx and ultimately cell swelling and plasma membrane rupture.

It has been shown that phosphorylation of MLKL 
A

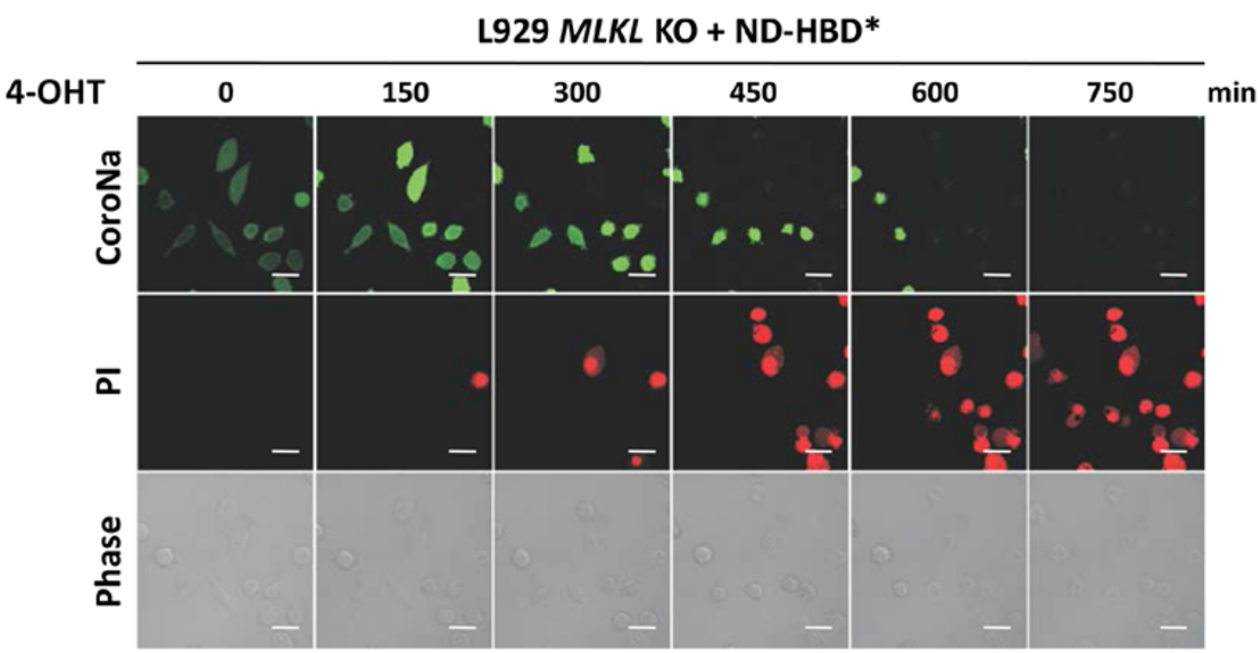

B

L929 MLKL KO + ND-HBD*

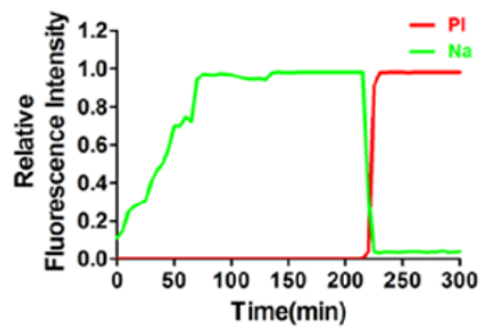

D

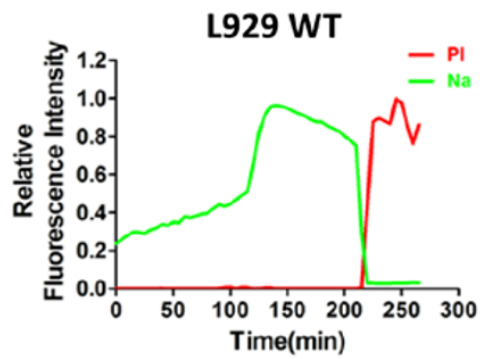

C

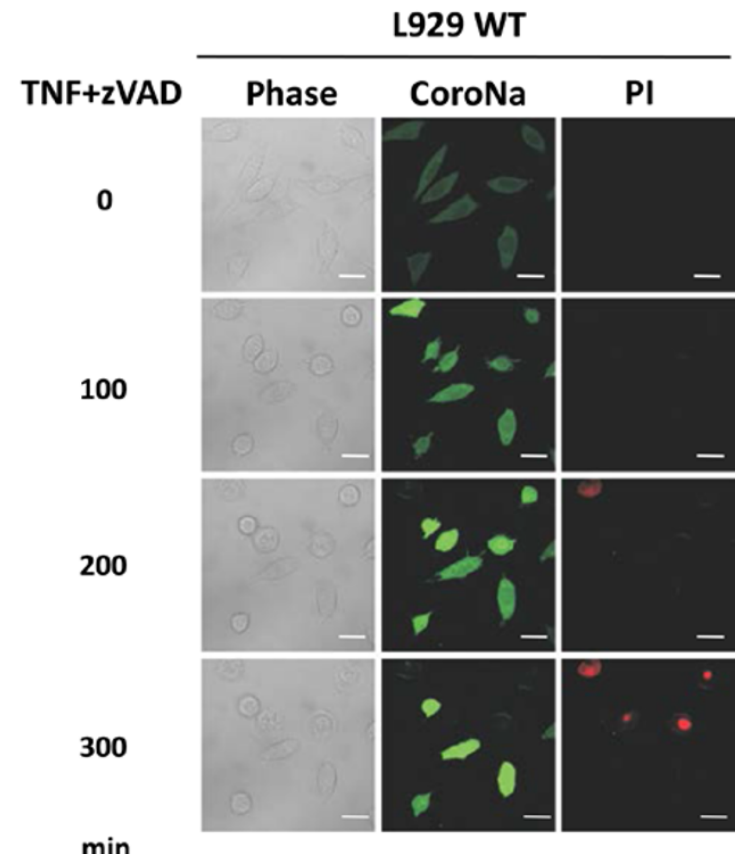

Figure 6 Sodium influx occurs before cell membrane breakage in necroptosis. (A) Representative time-lapse images of NDHBD*-expressing MLKL KO L929 cells undergoing necroptosis upon treatment of 4-OHT (1 $\mu \mathrm{M})$. The intracellular sodium concentration was measured by fluorescence sodium indicator CoroNa Green, and cell membrane integrity was monitored by PI uptake. Elapsed time is indicated above each frame and each scale bar represents $15 \mu \mathrm{m}$. (B) Relative changes in Coro$\mathrm{Na}$ Green and PI fluorescence over time in one representative cell shown in A were graphed. (C) Representative time-lapse images of L929 cells undergoing necroptosis induced by TNF + zVAD $(10 \mathrm{ng} / \mathrm{ml}+20 \mu \mathrm{M})$. Intracellular sodium concentration and PI uptake were monitored as described in A. Elapsed time is indicated on the left side of each frame and each scale bar represents $15 \mu \mathrm{m}$. (D) Relative changes in CoroNa Green and PI fluorescence over time in one representative cell shown in C were graphed.

on its kinase domain by RIP3 is required for MLKL to function in necroptosis [13]. As tetramerization of ND of MLKL is sufficient to trigger necroptosis, the phosphory- lation of MLKL by RIP3 is most likely to be required for the tetramerization of MLKL, but not the execution of cell death. RIP1, RIP3 and MLKL are the core com- 
A

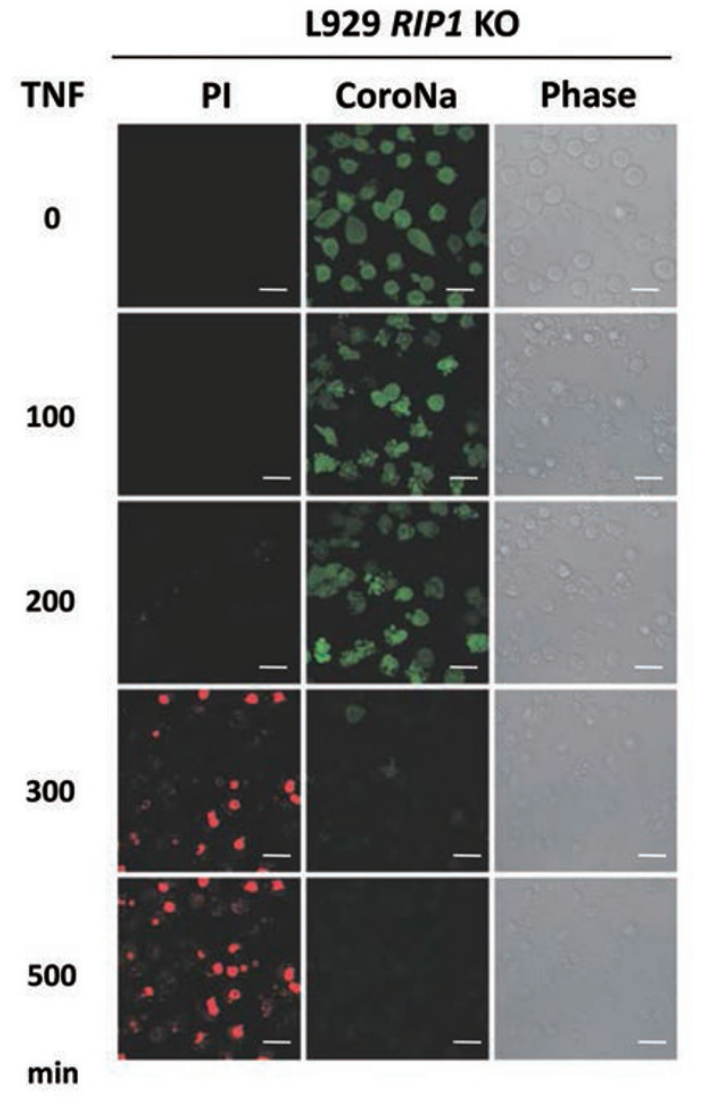

B

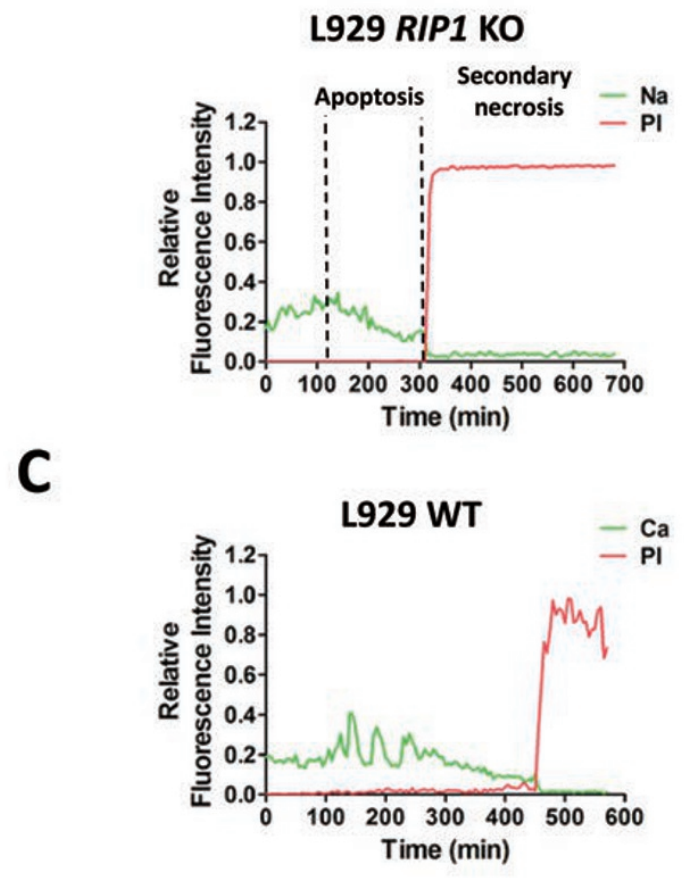

D

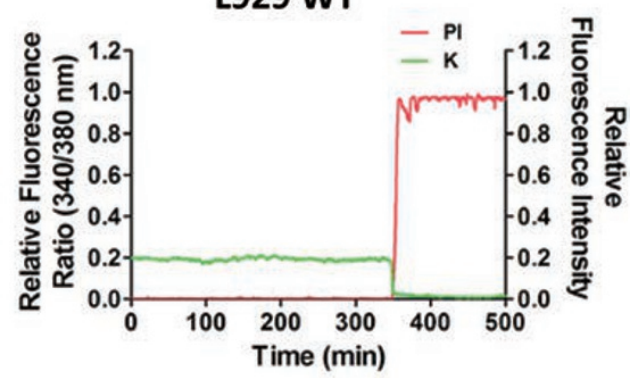

Figure 7 Sodium influx does not occur in the course of apoptosis, and potassium and calcium influx are not detected during necroptosis of L929 cells. (A) Representative time-lapse images of RIP1 KO L929 cells undergoing apoptosis induced by TNF $(10 \mathrm{ng} / \mathrm{ml})$. The intracellular sodium concentration was measured by fluorescence sodium indicator CoroNa Green and cell membrane integrity was monitored by PI uptake. Elapsed time is indicated on the left side of each frame and each scale bar represents $15 \mu \mathrm{m}$. (B) Relative changes in CoroNa Green and PI fluorescence over time in one representative cell shown in $\mathbf{A}$ were graphed. The stages of apoptosis and secondary necrosis of the selected single cell from $\mathbf{A}$ were indicated above dotted lines. (C) L929 cells loaded with calcium indicator Fluo-4, as described in Materials and Methods, were treated with TNF + zVAD $(10 \mathrm{ng} / \mathrm{ml}+20 \mu \mathrm{M})$. Relative changes in calcium indicator Fluo-4 and PI fluorescence over time in one representative cell were graphed. (D) Relative changes in potassium indicator PBFI and PI fluorescence over time in one representative L929 cell were graphed.

ponents of necrosome. Our data showed that RIP1 and RIP3 are co-translocated to lipid rafts (Figure 4E) and membrane fractions [29]. However, RIP1 and RIP3 may not interact with MLKL after the translocation to membrane, because mass spectrometry analysis of the chemically cross-linked MLKL in membrane fraction revealed only MLKL-MLKL interaction but not interactions of
MLKL and other proteins (data not shown). The MLKL complex on the plasma membrane could be multiple tetramers together because the cross-linked membrane MLKLs are larger than tetramers (data not shown). As RIP1 and RIP3 are not required for necroptosis triggered by forcing MLKL or its ND together (Figure 2C and data not shown), translocation of RIP1 and RIP3 to plasma 

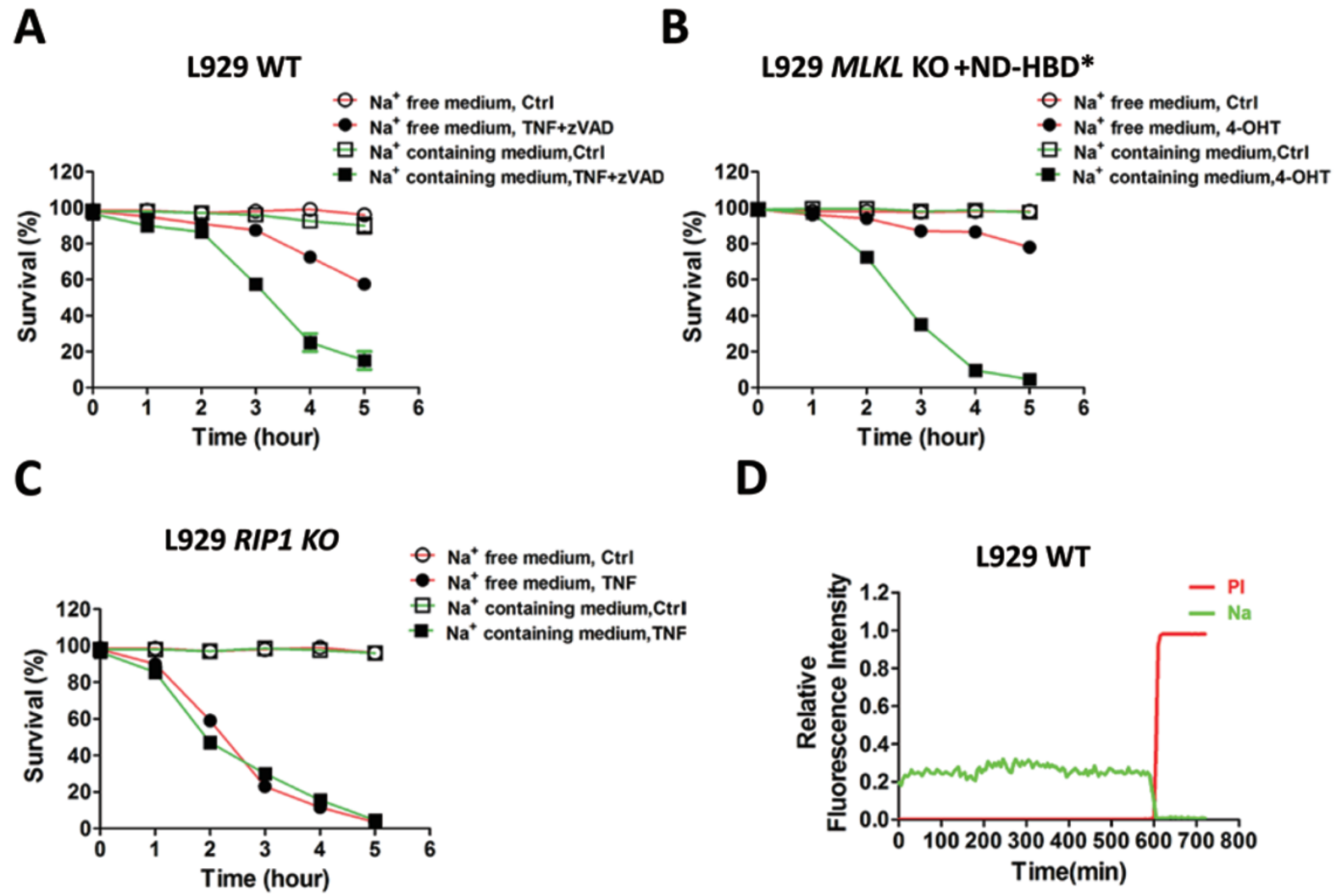

Figure 8 Sodium depletion inhibits necroptosis, but not apoptosis. (A) Viabilities of L929 cells treated with or without TNF + zVAD $(10 \mathrm{ng} / \mathrm{ml}+20 \mu \mathrm{M})$ in sodium-free or sodium-containing medium. (B) Viabilities of MLKL KO L929 cells expressing NDHBD* treated with or without 4-OHT $(1 \mu \mathrm{M})$ in sodium-free or sodium-containing medium. (C) Viabilities of RIP1 KO L929 cells treated with or without TNF $(10 \mathrm{ng} / \mathrm{ml})$ in sodium-free or sodium-containing medium. (D) L929 cells were treated with TNF + zVAD $(10 \mathrm{ng} / \mathrm{ml}+20 \mu \mathrm{M})$ in sodium-free medium, and relative changes in CoroNa Green and Pl fluorescence over time in one representative cell were graphed. Data shown represented the mean \pm SD of triplicates (A-C), and were representative of three independent experiments.

membrane is not required for necroptosis.

Pyroptosis is a caspase-1-dependent programmed cell death with morphology of cell size increase and loss of plasma membrane integrity, which resembles necrosis features [23]. It was proposed that formation of small ion-permeable pores in the plasma membrane mediates pyroptotic cell lysis $[25,26]$, because investigators were able to experimentally estimate that the pore size is 1.1-2.4 $\mathrm{nm}$ in diameter, and found that the addition of extracellular glycine or osmoprotectants of $2.4 \mathrm{~nm}$ or greater can prevent cell swelling and lysis. Despite the similarity in cell swelling and plasma membrane rupture, necroptosis appears to use a different mechanism because extracellular glycine and osmoprotectants such as PEG did not prevent TNF-induced necroptosis (data not shown). Moreover, ethidium bromide, which can pass the pore in pyroptotic cells, cannot be uptaken by necroptotic cells before plasma membrane rupture (data not shown). Thus, if there is pore forming or pore opening during the pro- cess of necroptosis, the pore should be smaller than that in pyroptotic cells.

The increase of sodium influx appears to be a common event after MLKL translocation to the plasma membrane, as 4-OHT-induced tetramerization of MLKL (ND) triggers quick necroptosis in all tested cell lines including HEK293T, HeLa, CHO and L929 cells. And the depletion of sodium from the culture medium inhibited the 4-OHT-induced cell death in all of these cell lines. However, we cannot exclude the possibility that the ion influx is not selective for sodium because it is the most abundant ion in the cell culture medium, and thus is the most visible one. We have tested the non-selective cation channel blocker flufenamic acid (FFA) and sodium channel blocker bupivacaine and did not find inhibitory effect of both drugs (data not shown). Involvement of ion channels or pumps in apoptosis has been reported. Deletion of the non-selective cation channel, transient receptor potential melastatin-like 7 (TRPM7), in primary mouse T 
cells blocked Fas-induced apoptosis [38]; hyperpolarization-activated cyclic nucleotide-gated channel 2 (HCN2)mediated prolonged $\mathrm{Ca}^{2+}$ uptake was shown to contribute to apoptosis-inducing factor (AIF)-induced apoptosis in lung carcinoma cells and primary culture of cortical neurons [39]; apoptosis of human Jurkat cells triggered by Fas ligand (FasL) or tumor necrosis factor-related apoptosis-inducing ligand (TRAIL), can be enhanced via treatment of ouabain, a $\mathrm{Na}^{+} / \mathrm{K}^{+}$-ATPase inhibitor [40]. Whether any of these ion channels participate in necroptosis is unclear. How MLKL functions in plasma membrane to regulate ion influx remains to be investigated.

\section{Materials and Methods}

\section{Cell culture}

HeLa, HEK293T, mouse fibrosarcoma L929 and Chinese hamster ovary $(\mathrm{CHO})$ cells were obtained from ATCC. RIP1 ${ }^{-}$, RIP3$^{-}$ and $M L K L^{-}$L929 cells were generated by TALEN method as previously described [41]. The KO cells were determined by the sequencing of targeted loci and immunoblotting of the expression of respective proteins. All cells were maintained in Dulbecco's modified Eagle's medium (DMEM), supplemented with $10 \%$ fetal bovine serum, $2 \mathrm{mM}$ L-glutamine, $100 \mathrm{IU}$ penicillin, and $100 \mathrm{mg} /$ $\mathrm{ml}$ streptomycin at $37^{\circ} \mathrm{C}$ in a humidified incubator containing $5 \%$ $\mathrm{CO}_{2}$.

\section{Reagents and antibodies}

Mouse TNF $\alpha$ was from eBioscience (San Diego, CA, USA). zVAD was obtained from Calbiochem. 4-hydroxytamoxifen (4$\mathrm{OHT}$ ) and propidium iodide (PI) were purchased from Sigma. All dyes including ion indicators (CoroNa Green, PBFI and Fluo-4), Mitotracker Red and DAPI were provided by Invitrogen (Carlsbad, CA, USA). Mouse anti-Flag antibodies (M2) were purchased from Sigma (Saint Louis, MO, USA). Mouse anti-HA (F-7), mouse anti- $\beta$-actin $(\mathrm{C} 4)$ and rabbit anti-CAV1 (N-20) antibodies were obtained from Santa Cruz Biotechnology, Inc. (Santa Cruz, CA, USA). Rabbit anti-FLOT antibodies were from ProteinTech, Inc. (Wuhan, Hubei). Mouse anti-RIP1 antibodies were obtained from BD Biosciences (San Jose, CA, USA). Anti-MLKL polyclonal antibodies were raised in rabbits using Escherichia coli-expressed GST-MLKL (100-200 amino acids). Anti-RIP3 antibodies were prepared as described previously [10].

\section{cDNA constructs and gene expression}

The mouse MLKL cDNA-encoding residues 1-464 was PCR amplified from a reverse-transcribed cDNA library derived from L929 cells. Full-length or truncated cDNAs of MLKL were cloned into BamHI and XhoI sites of the lentiviral vector pBOB using the Exo III-assisted ligase-free cloning method as described [42]. ND of MLKL fused with the HBD*, and its related mutants were created by cloning into the $\mathrm{pBOB}$ vector using standard PCR methods. HA-tagged mouse RIP3 expression vector was described previously [10]. All plasmids were verified by DNA sequencing. The details of the sequences are available upon request. For lentivirus production, HEK293T cells were transfected by the calcium phosphate precipitation method. The virus-containing medium was harvested 36-48 h later and was added to L929, HeLa or other cells as indicated with $10 \mu \mathrm{g} / \mathrm{ml}$ of polybrene. Infectious medium was changed $12 \mathrm{~h}$ later and cells were kept in culture until following analysis.

\section{Immunoprecipitation and western blotting}

Immunoprecipitation was performed using anti-Flag M2 or anti-HA beads as described [43, 44]. Western blotting of cell lysates and immunoprecipitates were performed, using anti-MLKL, anti-RIP3, anti-Flag, anti-HA, and other antibodies as indicated. For non-reducing SDS-PAGE, cells were washed with PBS once and lysed directly in loading buffer (150 mM Tris-HCl, 3\% SDS, $0.15 \%$ bromophenol blue and $15 \%$ glycerol, $\mathrm{pH} 6.8$ ) without $\beta$-mercaptoethanol.

\section{Cell viability assay}

Cell survival rates were determined by flow cytometry with two parameters: plasma membrane integrity and cell size. The plasma membrane integrity was tested by the ability of cells to exclude PI. Cells were trypsinized, collected by centrifugation, washed once with PBS, and resuspended in PBS containing $5 \mu \mathrm{g} / \mathrm{ml}$ PI. The levels of PI incorporation were quantified on a FACScan flow cytometer (EPICS XL; Beckman Coulter, Fullerton, CA, USA). Cell size was evaluated by forward-angle light scattering. PI-negative cells with a normal size were considered living. To explore the requirement of sodium for cell death execution, WT and RIPI KO L929 cells were cultured in sodium-free medium $(150 \mathrm{mM}$ NMDG, $2 \mathrm{mM} \mathrm{CaCl}$, $1 \mathrm{mM} \mathrm{MgCl}$, $10 \mathrm{mM}$ Hepes and $10 \mathrm{mM}$ Glucose, $\mathrm{pH}$ 7.3) or in normal medium by substituting NMDG with $\mathrm{NaCl}$. Necroptosis and apoptosis were triggered by addition of TNF $(10 \mathrm{ng} / \mathrm{ml})$ plus zVAD $(20 \mu \mathrm{M})$ and TNF $(10 \mathrm{ng} / \mathrm{ml})$ alone, respectively. Cells were counterstained with Hoechst (Beyotime, Shanghai) and PI. The survival rates were determined as the ratio of PI-negative cells to Hochest-positive cells.

\section{Lipid raft isolation}

Lipid rafts were isolated by sucrose density gradient centrifugation essentially as described [45]. In brief, WT L929 or MLKL KO L929 cells expressing ND-HBD* were treated with TNF plus zVAD or 4-OHT. All cells were lysed on ice for $20 \mathrm{~min}$ in $2 \mathrm{ml}$ of MNX buffer ( $1 \%$ Triton X-100 in $25 \mathrm{mM}$ MES, $150 \mathrm{mM} \mathrm{NaCl}$, $\mathrm{pH}$ 6.5) supplemented with protease inhibitor cocktail (Sigma) and homogenized (20 strokes) with a glass dounce homogenizer (HS Martin, Vineland, NJ, USA). The homogenates were mixed with $2 \mathrm{ml} \mathrm{90 \%} \mathrm{sucrose} \mathrm{made} \mathrm{with} \mathrm{MN} \mathrm{buffer} \mathrm{(without} \mathrm{Triton} \mathrm{X-100)}$ and placed on the bottom of a centrifuge tube. The samples were then overlaid with $4 \mathrm{ml}$ of $35 \%$ sucrose and $4 \mathrm{ml}$ of $5 \%$ sucrose. Gradients were centrifuged for $16 \mathrm{~h}$ at $175000 \times \mathrm{g}$. One milliliter fractions were collected from the top of the gradient and analyzed by western blotting.

\section{Confocal microscopy}

For live cell imaging, L929 or HeLa cells were seeded in 35mm glass bottom dishes (Nest, Shanghai) at a density of 1-3 $\times 10^{5}$ cells per well. To monitor the intracellular ion concentration, cells were loaded with sodium indicator CoroNa Green, potassium indicator PBFI and calcium indicator Fluo-4 according to the manufacturer's protocol. Necroptosis was initiated by treatment with TNF $(10 \mathrm{ng} / \mathrm{ml})$ and zVAD $(20 \mu \mathrm{M})$ to WT L929 cells or addition of 4-OHT $(1 \mu \mathrm{M})$ to $M L K L$ KO L929 cells reconstituted with ND-HBD*. Apoptosis was triggered through treatment with TNF 
alone to RIP1 KO L929 cells. PI ( $5 \mathrm{ng} / \mathrm{ml})$ was added to medium for monitoring cell-membrane integrity. Time-lapse images were acquired every 5 min for several hours depending on the cell death process. CoroNa Green and Fluo-4 fluorescence were excited under a $488 \mathrm{~nm}$ argon laser. Imaging was carried out using Zeiss LSM 780 with a $100 \times / 1.49$ NA oil objective at room temperature. PBFI fluorescence was measured by illuminating the cells with an alternating 340/380 light. Fluorescence intensity was measured at $510 \mathrm{~nm}$ by Nikon Elipse Ti-U inverted microscope with an optical filter changer (Sutter Instrument, Novato, CA, USA). Changes in intracellular potassium concentration were presented as the change in the ratio of fluorescence intensity for excitation at $340 \mathrm{~nm}$ and $380 \mathrm{~nm}$.

For fixed cell imaging, cells were fixed with freshly prepared $4 \%$ paraformaldehyde (PFA) in PBS. The fixed cells were then permeabilized in $0.2 \%$ Triton $\mathrm{X}-100 / \mathrm{PBS}$, blocked with $3 \%$ BSA in PBS, stained with anti-Flag (rabbit, 1:200, Sigma) or anti-HA (mouse, 1:100, Santa Cruz) and labeled with goat anti-mouse or rabbit AlexaFluor 488 (1:1 000, Invitrogen). Cells were counterstained with DAPI to visualize nuclei. All images were captured and processed using identical settings in the Zeiss LSM 780 laser scanning confocal microscope with a $100 \times / 1.49$ NA oil objective. Duplicate cultures were examined, and similar results were obtained in at least three independent experiments.

\section{TIRF and determination of bleaching steps}

Live cell TIRF imaging was carried out as described previously [35]. Briefly, HeLa cells expressing either HBD*-GFP or ND-HBD*-GFP were imaged in DMEM without phenol red (Invitrogen). Imaging was carried out using an OLYMPUS inverted microscope with a $100 \times / 1.49$ numerical aperture TIRF objective, equipped with through-the-objective TIRF illumination, a $37{ }^{\circ} \mathrm{C}$ temperature-controlled stage and an objective warmer (Live Cell Instrument, Seoul, Korea). A 488 nm argon laser (Andor, Belfast, Northern Ireland) was used as light source. Images were acquired with an EMCCD camera (Andor, Belfast, Northern Ireland) driven by iQ software (Andor).

To determine the stoichiometry of ND-HBD* complex, SiMPull experiments were performed as described previously [33, 46]. Briefly, glass coverslips were sonicated, rinsed extensively with hot water and dried in a $\mathrm{N}_{2}$ stream. PEG passivation of microscope slides was performed by applying $100 \mu \mathrm{l}$ of $2 \mathrm{mg} / \mathrm{ml} \mathrm{mPEG-silane}$ MW 2000 and $2 \mu \mathrm{g} / \mathrm{ml}$ biotin-PEG-silane MW 3400 (both from Laysan Bio, Arab, AL, USA) in $80 \%$ ethanol brought to $\mathrm{pH} 2.0$ with $\mathrm{HCl}$, and then dried by baking at $70{ }^{\circ} \mathrm{C}$ for $16 \mathrm{~h}$. HeLa cell extracts containing either HBD*-GFP-Flag or ND-HBD*-GFPFlag were captured by surface-tethered anti-Flag antibodies after several rounds of washing. Immobile single fluorescent molecules were visualized by an objective-type TIRF microscope. Movies of 400-1 000 frames were acquired at frame rates of $10 \mathrm{~Hz}$ with a back-illuminated EMCCD camera. To determine the time course of the emission intensity from a fluorescent spot, a Gaussian profile with a FWHM of 6 pixels around the center position was fitted to the raw images. We discarded the traces that showed emission intensities with no clear bleaching steps, and manually counted the number of bleaching steps.

\section{Image analysis and statistical analysis}

Images were saved as 16-bit TIFF files. Quantitative image analysis was carried out on unprocessed images using ImageJ software. For estimating changes in ion indicators fluorescence over time in confocal images, individual cells were selected manually and fluorescence values were measured over the entire stack. For each condition, at least two independent experiments were run and analyzed. Absent error bars in graphs signify SD values smaller than the graphic symbols.

\section{Acknowledgments}

This work was supported by the National Basic Research Program of China (973 Program; 2013CB944903 and 2014CB541804), the National Natural Science Foundation of China (31330047, 91029304 and 31221065), the Hi-Tech Research and Development Program of China (863 program; 2012AA02A201), the 111 Project (B12001), and the Open Research Fund of State Key Laboratory of Cellular Stress Biology, Xiamen University (SKLCSB2012KF003).

\section{References}

1 Laster SM, Wood JG, Gooding LR. Tumor necrosis factor can induce both apoptic and necrotic forms of cell lysis. J Immunol 1988; 141:2629-2634.

2 Vandenabeele P, Galluzzi L, Vanden Berghe T, Kroemer G. Molecular mechanisms of necroptosis: an ordered cellular explosion. Nat Rev Mol Cell Biol 2010; 11:700-714.

3 Christofferson DE, Yuan J. Necroptosis as an alternative form of programmed cell death. Curr Opin Cell Biol 2010; 22:263268.

4 Degterev A, Huang Z, Boyce M, et al. Chemical inhibitor of nonapoptotic cell death with therapeutic potential for ischemic brain injury. Nat Chem Biol 2005; 1:112-119.

5 Haas TL, Emmerich CH, Gerlach B, et al. Recruitment of the linear ubiquitin chain assembly complex stabilizes the TNFR1 signaling complex and is required for TNF-mediated gene induction. Mol Cell 2009; 36:831-844.

6 Bertrand MJ, Milutinovic S, Dickson KM, et al. cIAP1 and cIAP2 facilitate cancer cell survival by functioning as E3 ligases that promote RIP1 ubiquitination. Mol Cell 2008; 30:689-700.

7 Hacker H, Karin M. Regulation and function of IKK and IKKrelated kinases. Sci STKE 2006; 357:re13.

8 Micheau O, Tschopp J. Induction of TNF receptor I-mediated apoptosis via two sequential signaling complexes. Cell 2003; 114:181-190.

9 Vandenabeele P, Declercq W, Van Herreweghe F, Vanden Berghe T. The role of the kinases RIP1 and RIP3 in TNF-induced necrosis. Sci Signal 2010; 3:re4.

10 Zhang DW, Shao J, Lin J, et al. RIP3, an energy metabolism regulator that switches TNF-induced cell death from apoptosis to necrosis. Science 2009; 325:332-336.

11 Cho Y, Challa S, Moquin D, et al. Phosphorylation-driven assembly of the RIP1-RIP3 complex regulates programmed necrosis and virus-induced inflammation. Cell 2009; 137:11121123 .

12 He S, Wang L, Miao L, et al. Receptor interacting protein kinase-3 determines cellular necrotic response to TNF-alpha. Cell 2009; 137:1100-1111. 
13 Sun L, Wang H, Wang Z, et al. Mixed lineage kinase domainlike protein mediates necrosis signaling downstream of RIP3 kinase. Cell 2012; 148:213-227.

14 Zhao J, Jitkaew S, Cai Z, et al. Mixed lineage kinase domainlike is a key receptor interacting protein 3 downstream component of TNF-induced necrosis. Proc Natl Acad Sci USA 2012; 109:5322-5327.

15 Li J, McQuade T, Siemer A, et al. The RIP1/RIP3 necrosome forms a functional amyloid signaling complex required for programmed necrosis. Cell 2012; 150:339-350.

16 Remillard CV, Yuan JX. Activation of $\mathrm{K}^{+}$channels: an essential pathway in programmed cell death. Am J Physiol Lung Cell Mol Physiol 2004; 286:L49-L67.

17 Lande MB, Donovan JM, Zeidel ML. The relationship between membrane fluidity and permeabilities to water, solutes, ammonia and protons. J Gen Physiol 1995; 106:67-84.

18 Erecinska M, Silver IA. ATP and brain function. J Cereb Blood Flow Metab 1989; 9:2-19.

19 Xiao AY, Wei L, Xia S, Rothman S, Yu SP. Ionic mechanism of ouabain-induced concurrent apoptosis and necrosis in individual cultured cortical neurons. J Neurosci 2002; 22:13501362.

$20 \mathrm{Yu}$ SP, Yeh CH, Sensi SL, et al. Mediation of neuronal apoptosis by enhancement of outward potassium current. Science 1997; 278:114-117.

21 Bortner CD, Cidlowski JA. Uncoupling cell shrinkage from apoptosis reveals that $\mathrm{Na}^{+}$influx is required for volume loss during programmed cell death. J Biol Chem 2003; 278:3917639184.

22 McCarthy JV, Cotter TG. Cell shrinkage and apoptosis: a role for potassium and sodium ion efflux. Cell Death Differ 1997; 4:756-770.

23 Bergsbaken T, Fink SL, Cookson BT. Pyroptosis: host cell death and inflammation. Nat Rev Microbiol 2009; 7:99-109.

24 Sun GW, Lu J, Pervaiz S, Cao WP, Gan YH. Caspase-1 dependent macrophage death induced by Burkholderia pseudomallei. Cell Microbiol 2005; 7:1447-1458.

25 Fink SL, Cookson BT. Caspase-1-dependent pore formation during pyroptosis leads to osmotic lysis of infected host macrophages. Cell Microbiol 2006; 8:1812-1825.

26 Fink SL, Bergsbaken T, Cookson BT. Anthrax lethal toxin and Salmonella elicit the common cell death pathway of caspase1-dependent pyroptosis via distinct mechanisms. Proc Natl Acad Sci USA 2008; 105:4312-4317.

27 van der Velden AW, Velasquez M, Starnbach MN. Salmonella rapidly kill dendritic cells via a caspase-1-dependent mechanism. J Immunol 2003; 171:6742-6749.

28 Zhang DW, Zheng M, Zhao J, et al. Multiple death pathways in TNF-treated fibroblasts: RIP3- and RIP1-dependent and independent routes. Cell Res 2011; 21:368-371.

29 Chen W, Zhou Z, Li L, et al. Diverse sequence determinants control human and mouse receptor interacting protein 3 (RIP3) and mixed lineage kinase domain-like (MLKL) interaction in necroptotic signaling. J Biol Chem 2013; 288:16247-16261.

30 Zhou Z, Han V, Han J. New components of the necroptotic pathway. Protein Cell 2012; 3:811-817.

31 Brandt ME, Vickery LE. Cooperativity and dimerization of recombinant human estrogen receptor hormone-binding domain. J Biol Chem 1997; 272:4843-4849.
32 Gallinari P, Lahm A, Koch U, et al. A functionally orthogonal estrogen receptor-based transcription switch specifically induced by a nonsteroid synthetic ligand. Chem Biol 2005; 12:883-893.

33 Jain A, Liu R, Xiang YK, Ha T. Single-molecule pull-down for studying protein interactions. Nat Protoc 2012; 7:445-452.

34 Stenkula KG, Lizunov VA, Cushman SW, Zimmerberg J. Insulin controls the spatial distribution of GLUT4 on the cell surface through regulation of its postfusion dispersal. Cell Metab 2010; 12:250-259.

$35 \mathrm{Ji} \mathrm{W}, \mathrm{Xu}$ P, Li Z, et al. Functional stoichiometry of the unitary calcium-release-activated calcium channel. Proc Natl Acad Sci USA 2008; 105:13668-13673.

36 Xie T, Peng W, Yan C, Wu J, Gong X, Shi Y. Structural insights into RIP3-mediated necroptotic signaling. Cell Rep 2013; 5:1-9.

37 Murphy JM, Czabotar PE, Hildebrand JM, et al. The pseudokinase MLKL mediates necroptosis via a molecular switch mechanism. Immunity 2013; 39:443-453.

38 Desai BN, Krapivinsky G, Navarro B, et al. Cleavage of TRPM7 releases the kinase domain from the ion channel and regulates its participation in Fas-induced apoptosis. Dev Cell 2012; 12:1149-1162.

39 Norberg E, Karlsson M, Korenovska O, et al. Critical role for hyperpolarization-activated cyclic nucleotide-gated channel 2 in the AIF-mediated apoptosis. EMBO J 2010; 29:3869-3878.

40 Panayiotidis MI, Franco R, Bortner CD, Cidlowski JA. Ouabain-induced perturbations in intracellular ionic homeostasis regulate death receptor-mediated apoptosis. Apoptosis 2010; 15:834-849.

41 Zhang F, Cong L, Lodato S, Kosuri S, Church GM, Arlotta P. Efficient construction of sequence-specific TAL effectors for modulating mammalian transcription. Nat Biotechnol 2011; 29:149-153.

42 Li C, Evans RM. Ligation independent cloning irrespective of restriction site compatibility. Nucleic Acids Res 1997; 25:41654166.

43 Zhou H, Zheng M, Chen J, et al. Determinants that control the specific interactions between TAB1 and p38alpha. Mol Cell Biol 2006; 26:3824-3834.

44 Li Q, Zhang N, Zhang D, et al. Determinants that control the distinct subcellular localization of p38alpha-PRAK and p38beta-PRAK complexes. J Biol Chem 2008; 283:11014-11023.

45 Legler DF, Micheau O, Doucey MA, Tschopp J, Born C. Recruitment of TNF receptor 1 to lipid rafts is essential for $\mathrm{TNF} \alpha$-mediated NF- $\mathrm{\kappa B}$ activation. Immunity 2003; 18:655664.

46 Breitsprecher D, Jaiswal R, Bombardier JP, Gould CJ, Gelles J, Goode BL. Rocket launcher mechanism of collaborative actin assembly defined by single-molecule imaging. Science 2012; 336:1164-1168.

(Supplementary information is linked to the online version of the paper on the Cell Research website.)

This work is licensed under the Creative Commons Attribution-NonCommercial-No Derivative Works
3.0 Unported License. To view a copy of this license, visit http:// creativecommons.org/licenses/by-nc-nd/3.0 